\title{
PKS 2005-489 at VHE: four years of monitoring with HESS and simultaneous multi-wavelength observations
}

HESS Collaboration, F. Acero ${ }^{15}$, F. Aharonian ${ }^{1,13}$, A. G. Akhperjanian ${ }^{2}$, G. Anton ${ }^{16}$, U. Barres de Almeida ${ }^{8, \star}$, A. R. Bazer-Bachi ${ }^{3}$, Y. Becherini ${ }^{12}$, B. Behera ${ }^{14}$, W. Benbow ${ }^{1, \star \star}$, K. Bernlöhr ${ }^{1,5}$, A. Bochow ${ }^{1}$, C. Boisson ${ }^{6}$, J. Bolmont ${ }^{19}$, V. Borrel ${ }^{3}$, J. Brucker ${ }^{16}$, F. Brun ${ }^{19}$, P. Brun ${ }^{7}$, R. Bühler ${ }^{1}$, T. Bulik ${ }^{29}$, I. Büsching ${ }^{9}$, T. Boutelier ${ }^{17}$, P. M. Chadwick ${ }^{8}$, A. Charbonnier ${ }^{19}$, R. C. G. Chaves $^{1}$, A. Cheesebrough ${ }^{8}$, L.-M. Chounet ${ }^{10}$, A. C. Clapson ${ }^{1}$, G. Coignet ${ }^{11}$, L. Costamante ${ }^{1,30, \star \star \star}$, M. Dalton ${ }^{5}$, M. K. Daniel ${ }^{8}$, I. D. Davids ${ }^{22,9}$, B. Degrange ${ }^{10}$, C. Deil $^{1}$, H. J. Dickinson ${ }^{8}$, A. Djannati-Atai ${ }^{12}$, W. Domainko ${ }^{1}$, L. O’C. Drury ${ }^{13}$, F. Dubois ${ }^{11}$, G. Dubus ${ }^{17}$, J. Dyks ${ }^{24}$, M. Dyrda ${ }^{28}$, K. Egberts ${ }^{1}$, P. Eger ${ }^{16}$, P. Espigat ${ }^{12}$, L. Fallon ${ }^{13}$, C. Farnier ${ }^{15}$, S. Fegan ${ }^{10}$, F. Feinstein ${ }^{15}$, A. Fiasson ${ }^{11}$, A. Förster ${ }^{1}$, G. Fontaine ${ }^{10}$, M. Füßling ${ }^{5}$, S. Gabici ${ }^{13}$, Y. A. Gallant ${ }^{15}$, L. Gérard ${ }^{12}$, D. Gerbig ${ }^{21}$, B. Giebels ${ }^{10}$, J. F. Glicenstein ${ }^{7}$, B. Glück ${ }^{16}$, P. Goret ${ }^{7}$, D. Göring ${ }^{16}$, M. Hauser ${ }^{14}$, S. Heinz ${ }^{16}$, G. Heinzelmann ${ }^{4}$, G. Henri ${ }^{17}$, G. Hermann ${ }^{1}$, J. A. Hinton ${ }^{25}$, A. Hoffmann ${ }^{18}$, W. Hofmann ${ }^{1}$, P. Hofverberg ${ }^{1}$, M. Holleran ${ }^{9}$, S. Hoppe ${ }^{1}$, D. Horns ${ }^{4}$, A. Jacholkowska ${ }^{19}$, O. C. de Jager ${ }^{9}$, C. Jahn ${ }^{16}$, I. Jung ${ }^{16}$, K. Katarzyński ${ }^{27}$, U. Katz ${ }^{16}$, S. Kaufmann ${ }^{14}$, M. Kerschhaggl ${ }^{5}$, D. Khangulyan ${ }^{1}$, B. Khélifi ${ }^{10}$, D. Keogh ${ }^{8}$, D. Klochkov ${ }^{18}$, W. Kluźniak ${ }^{24}$, T. Kneiske ${ }^{4}$, Nu. Komin ${ }^{7}$, K. Kosack ${ }^{1}$, R. Kossakowski ${ }^{11}$, G. Lamanna ${ }^{11}$, J.-P. Lenain ${ }^{6}$, T. Lohse ${ }^{5}$, V. Marandon ${ }^{12}$, O. Martineau-Huynh ${ }^{19}$, A. Marcowith ${ }^{15}$, J. Masbou ${ }^{11}$, D. Maurin ${ }^{19}$, T. J. L. McComb ${ }^{8}$, M. C. Medina ${ }^{6}$, J. Méhault ${ }^{15}$, R. Moderski ${ }^{24}$, E. Moulin ${ }^{7}$, M. Naumann-Godo ${ }^{10}$, M. de Naurois ${ }^{19}$, D. Nedbal ${ }^{20}$, D. Nekrassov ${ }^{1}$, B. Nicholas ${ }^{26}$, J. Niemiec $^{28}$, S. J. Nolan ${ }^{8}$, S. Ohm ${ }^{1}$, J.-F. Olive ${ }^{3}$, E. de Oña Wilhelmi ${ }^{1}$, K. J. Orford ${ }^{8}$, M. Ostrowski ${ }^{23}$, M. Panter ${ }^{1}$, M. Paz Arribas ${ }^{5}$, G. Pedaletti ${ }^{14}$, G. Pelletier ${ }^{17}$, P.-O. Petrucci ${ }^{17}$, S. Pita ${ }^{12}$, G. Pühlhofer ${ }^{18,14}$, M. Punch ${ }^{12}$, A. Quirrenbach ${ }^{14}$,

B. C. Raubenheimer ${ }^{9}$, M. Raue ${ }^{1,30}$, S. M. Rayner ${ }^{8}$, M. Renaud ${ }^{12,1}$, F. Rieger ${ }^{1,30}$, J. Ripken ${ }^{4}$, L. Rob ${ }^{20}$, S. Rosier-Lees $^{11}$, G. Rowell ${ }^{26}$, B. Rudak ${ }^{24}$, C. B. Rulten ${ }^{8}$, J. Ruppel ${ }^{21}$, V. Sahakian ${ }^{2}$, A. Santangelo ${ }^{18}$, R. Schlickeiser ${ }^{21}$, F. M. Schöck ${ }^{16}$, U. Schwanke ${ }^{5}$, S. Schwarzburg ${ }^{18}$, S. Schwemmer ${ }^{14}$, A. Shalchi ${ }^{21}$, M. Sikora ${ }^{24}$, J. L. Skilton ${ }^{25}$, H. Sol ${ }^{6}$, Ł. Stawarz ${ }^{23}$,

R. Steenkamp ${ }^{22}$, C. Stegmann ${ }^{16}$, F. Stinzing ${ }^{16}$, G. Superina ${ }^{10}$, A. Szostek ${ }^{23,17}$, P. H. Tam ${ }^{14}$, J.-P. Tavernet ${ }^{19}$, R. Terrier ${ }^{12}$, O. Tibolla ${ }^{1}$, M. Tluczykont ${ }^{4}$, C. van Eldik ${ }^{1}$, G. Vasileiadis ${ }^{15}$, C. Venter ${ }^{9}$, L. Venter $^{6}$, J. P. Vialle $^{11}$, P. Vincent ${ }^{19}$, M. Vivier ${ }^{7}$, H. J. Völk ${ }^{1}$, F. Volpe ${ }^{1}$, S. J. Wagner ${ }^{14}$, M. Ward ${ }^{8}$, A. A. Zdziarski ${ }^{24}$, and A. Zech ${ }^{6}$

(Affiliations can be found after the references)

Received 6 August 2009 / Accepted 10 November 2009

\section{ABSTRACT}

Aims. Our aim is to study the very high energy (VHE; $E>100 \mathrm{GeV}$ ) $\gamma$-ray emission from BL Lac objects and the evolution in time of their broad-band spectral energy distribution (SED).

Methods. VHE observations of the high-frequency peaked BL Lac object PKS 2005-489 were made with the High Energy Stereoscopic System (HESS) from 2004 through 2007. Three simultaneous multi-wavelength campaigns at lower energies were performed during the HESS data taking, consisting of several individual pointings with the XMM-Newton and RXTE satellites.

Results. A strong VHE signal, $\sim 17 \sigma$ total, from PKS 2005-489 was detected during the four years of HESS observations (90.3 h live time). The integral flux above the average analysis threshold of $400 \mathrm{GeV}$ is $\sim 3 \%$ of the flux observed from the Crab Nebula and varies weakly on time scales from days to years. The average VHE spectrum measured from $\sim 300 \mathrm{GeV}$ to $\sim 5 \mathrm{TeV}$ is characterized by a power law with a photon index, $\Gamma=3.20 \pm 0.16_{\text {stat }} \pm 0.10_{\text {syst }}$. At X-ray energies the flux is observed to vary by more than an order of magnitude between 2004 and 2005 . Strong changes in the X-ray spectrum $\left(\Delta \Gamma_{X} \approx 0.7\right)$ are also observed, which appear to be mirrored in the VHE band.

Conclusions. The SED of PKS 2005-489, constructed for the first time with contemporaneous data on both humps, shows significant evolution. The large flux variations in the X-ray band, coupled with weak or no variations in the VHE band and a similar spectral behavior, suggest the emergence of a new, separate, harder emission component in September 2005.

Key words. galaxies: active - BL Lacertae objects: individual: PKS 2005-489 - gamma rays: galaxies - X-rays: individuals: PKS 2005-489

\footnotetext{
* Supported by CAPES Foundation, Ministry of Education of Brazil. $\star \star$ Now at Harvard-Smithsonian Center for Astrophysics, Cambridge, USA.

$\star \star \star$ Now at W.W. Hansen Experimental Physics Laboratory \& Kavli Institute for Particle Astrophysics and Cosmology, Stanford University, Stanford, USA.
}

\section{Introduction}

PKS 2005-489 is one of the brightest BL Lac objects, at all wavelengths, in the Southern Hemisphere. It was initially discovered as a strong radio source in the Parkes $2.7 \mathrm{GHz}$ survey (Wall et al. 1975) and later identified as a BL Lac object (Wall et al. 1986). It belongs to the complete 1-Jy radio catalog 
(Stickel et al. 1991), and is one of the few extragalactic objects detected in the EUV band (Marshall et al. 1995). Its redshift, $z=0.071$ (Falomo et al. 1987), is determined from weak, narrow emission lines observed during a low optical state.

PKS 2005-489 is classified as a High-frequency peaked BL Lac object (HBL; Giommi \& Padovani 1994), because of its high X-ray-to-radio flux ratio (Sambruna et al. 1995) and because its broad-band spectral energy distribution (SED) peaks in the UV-soft X-ray band. As is typical of HBLs, the X-ray spectrum is dominated by the synchrotron emission of highenergy electrons. The second SED hump is expected to peak in the $\mathrm{GeV}-\mathrm{TeV} \gamma$-ray band, and is commonly believed to be produced by the same electrons up-scattering via the inverse Compton mechanism seed photons of lower energy.

In the X-ray band, PKS 2005-489 has been studied extensively, showing an extreme flux and spectral variability. Large flux variations with correlated spectral hardening of the generally steep spectrum (photon index ${ }^{1} \Gamma=2.7-3.1$ ) were observed during five EXOSAT observations (Giommi et al. 1990; Sambruna et al. 1994). Two ROSAT observations in 1992 confirmed the EXOSAT results and similarly show a soft spectrum $(\Gamma \simeq 3$; Sambruna et al. 1995). A harder spectrum $(\Gamma=2.3$ from 2 to $10 \mathrm{keV}$ ) was observed in September 1996, during BeppoSAX observations of a brighter X-ray state (Padovani et al. 2001). In October-November 1998 PKS 2005-489 underwent a period of exceptional activity, with several strong X-ray flares. RXTE monitoring observations (Perlman et al. 1999) were performed during the entire epoch, and the $2-10 \mathrm{keV}$ flux reached $\sim 3 \times 10^{-10} \mathrm{erg} \mathrm{cm}^{-2} \mathrm{~s}^{-1}$, approximately 30 times higher than the ROSAT values. These RXTE observations yielded a detection up to $40 \mathrm{keV}$ and showed variations in the photon index between $\Gamma=2.3$ and 2.8. An X-ray flare alert also triggered BeppoSAX observations on November 1-2, 1998. The X-ray spectrum was measured between 0.1 to $200 \mathrm{keV}$ and was characterized by a curved shape and harder photon indices, in both the soft $\left(\Gamma_{1}=2.0\right)$ and hard $\left(\Gamma_{2}=2.2\right) \mathrm{X}$-ray bands $(<$ and $>2 \mathrm{keV}$; Tagliaferri et al. 2001). More recently, observations with the Swift satellite have generally shown PKS 2005-489 in a lowflux, steep-spectrum state ( $\Gamma \simeq 3$, Massaro et al. 2008).

Strong correlations between $\mathrm{X}$-ray and $\gamma$-ray emission have been observed in many HBL (e.g. Pian et al. 1998; Maraschi et al. 1999; Krawczynski et al. 2004; Fossati et al. 2008; Aharonian et al. 2009), and are typically expected in a synchrotron-Compton scenario. Therefore the very bright flux and large flux/spectral variability at X-ray energies make PKS 2005-489 one of the most promising targets for observing a similar behavior in the $\gamma$-ray domain.

In the VHE $(E>100 \mathrm{GeV})$ band, PKS 2005-489 was detected neither during observations made between 1993 and 2000 by either the CANGAROO or Durham groups (Roberts et al. 1998, 1999; Nishijima 2002; Chadwick et al. 2000), nor by HESS with a partial array during its commissioning in 2003. In 2004, HESS discovered VHE $\gamma$-ray emission from PKS 2005-489 with a significance of $6.7 \sigma$, at a flux of a few percent of the Crab Nebula (Aharonian et al. 2005). The measured spectrum was soft $(\Gamma=4.0)$. In the $\mathrm{MeV}-\mathrm{GeV}$ band, PKS 2005-489 is one of the few HBL detected by EGRET. However, the observed significance is marginal: $3.7 \sigma$ above $100 \mathrm{MeV}$ (Lin et al. 1996) and $4.1 \sigma$ above $1 \mathrm{GeV}$ (Lamb \& Macomb 1997). It was instead detected by Fermi with high significance $(>10 \sigma)$, during the first three months of operation (2008, Aug.-Oct., Abdo et al. 2009).

1 The power-law spectrum is described as $N(E)=N_{0} E^{-\Gamma}$.
Because of the high potential for strong $\gamma$-ray activity, PKS 2005-489 has been monitored at VHE by HESS every year since its detection in 2004. Several campaigns of coordinated observations with the X-ray satellites XMM-Newton and RXTE were also performed. These simultaneous observations, sampling the same particle distribution through two different emission processes, represent a powerful diagnostic tool for probing the conditions of the inner blazar jet, especially during flaring events (Coppi \& Aharonian 1999).

In this article the results of all HESS observations taken from 2004 through 2007 are presented, together with the results of the multi-wavelength observations. These campaigns characterize the SED of PKS 2005-489 during different states and, for the first time, sample both humps of the SED simultaneously. A re-analysis of the 2004 HESS data is also provided, which benefits from an improved calibration of the absolute energy scale with respect to the previously published result.

\section{HESS observations and analysis technique}

PKS 2005-489 was observed with the HESS array (Hinton 2004) for a total of $158.0 \mathrm{~h}$ (352 runs of $28 \mathrm{~min}$ each) from 2004 through 2007. During these observations the array tracked a position offset from the blazar by $0.5^{\circ}$ in alternating directions to enable both on-source observations and simultaneous estimation of the background induced by charged cosmic rays. A total of 207 runs pass the standard HESS data-quality selection, yielding an exposure of $90.3 \mathrm{~h}$ live time at a mean zenith angle $Z_{\text {mean }}=35^{\circ}$. The results presented here were generated using the standard HESS calibration methods (Aharonian et al. 2004) and analysis tools (Benbow 2005), with the standard cuts event-selection criteria (except for the 2007 spectrum, see Sect. 3.2). On-source data were taken from a circular region of radius $\theta_{\text {cut }}=0.11^{\circ}$ centered on PKS 2005-489, and the background (off-source data) was estimated using the ReflectedRegion method (Berge et al. 2007). Equation (17) in Li \& Ma (1983) was used to calculate the significance of any excess. All VHE integral fluxes reported throughout this article were calculated assuming the time-average photon index of $\Gamma=3.20$ determined in Sect. 3.2.

The PKS 2005-489 observations presented here span four years (2004-2007) of HESS data taking. During this time the optical throughput of the instrument decreased, because of the degradation of the reflective surfaces of the mirrors and Winston cones, as well as accumulation of dust on the optical elements. For the entire data sample, the optical efficiency has decreased by an average of $28 \%$ compared to a newly commissioned instrument, with its mirrors installed in Oct. 2001, Dec. 2002, June 2003, and August 2003 on CT3, CT2, CT4, and CT1, respectively. To minimize the effects of long-term variation in the optical efficiency of the HESS array, the estimated energy of each event was corrected using the ratio of efficiencies determined on a run-wise basis from simulated and observed muons (Aharonian et al. 2006a). After accounting for the decreasing optical throughput of the HESS array, the average energy threshold of the analysis at $Z_{\text {mean }}$ is $400 \mathrm{GeV}$.

\section{HESS results}

PKS 2005-489 was clearly detected in each of the four years (2004-2007) that it was observed by HESS. A total of 1233 excess events, corresponding to a statistical significance of 16.7 standard deviations $(\sigma)$, was detected from the direction 
The HESS Collaboration: VHE monitoring of PKS 2005-489 and simultaneous multi-wavelength observations

Table 1. Results from long-term HESS observations of PKS 2005-489.

\begin{tabular}{ccccccccccccc}
\hline \hline $\begin{array}{c}\text { Dark } \\
\text { period }\end{array}$ & $\begin{array}{c}\text { MJD } \\
\text { first }\end{array}$ & $\begin{array}{c}\text { MJD } \\
\text { last }\end{array}$ & $\begin{array}{c}\text { Time } \\
{[\mathrm{h}]}\end{array}$ & On & Off & $\alpha$ & Excess & $\begin{array}{c}\text { Sig } \\
{[\sigma]}\end{array}$ & $\begin{array}{c}I(>400 \mathrm{GeV})^{a} \\
{\left[10^{-12} \mathrm{~cm}^{-2} \mathrm{~s}^{-1}\right]}\end{array}$ & $\begin{array}{c}\mathrm{Crab}^{b} \\
\%\end{array}$ & $\chi^{2}\left(\mathrm{NDF}^{c}\right)$ & $P_{\left(\chi^{2}\right)^{c}}$ \\
\hline $06 / 2004$ & 53171 & 53185 & 8.1 & 678 & 5877 & 0.0919 & 138 & 5.4 & $2.64 \pm 0.50$ & 3.0 & $7.2(10)$ & 0.71 \\
$07 / 2004$ & 53199 & 53205 & 1.5 & 105 & 985 & 0.0909 & 15 & 1.5 & $1.06 \pm 1.18$ & 1.2 & $1.4(1)$ & 0.75 \\
$09 / 2004$ & 53255 & 53268 & 5.6 & 342 & 3171 & 0.0916 & 52 & 2.8 & $1.89 \pm 0.78$ & 2.1 & $2.1(5)$ & 0.84 \\
$10 / 2004$ & 53285 & 53292 & 9.0 & 569 & 5065 & 0.0916 & 105 & 4.5 & $2.58 \pm 0.62$ & 2.9 & $1.5(4)$ & 0.83 \\
$07 / 2005$ & 53582 & 53595 & 9.4 & 573 & 4504 & 0.0908 & 164 & 7.3 & $3.56 \pm 0.67$ & 4.0 & $9.2(9)$ & 0.42 \\
$08 / 2005$ & 53609 & 53618 & 5.1 & 286 & 2159 & 0.0989 & 73 & 4.5 & $2.86 \pm 0.93$ & 3.2 & $4.6(4)$ & 0.33 \\
$09 / 2005$ & 53639 & 53646 & 18.1 & 1072 & 9125 & 0.0928 & 225 & 7.1 & $2.93 \pm 0.46$ & 3.3 & $13.1(6)$ & 0.041 \\
$06 / 2006$ & 53908 & 53909 & 1.3 & 127 & 824 & 0.0938 & 50 & 4.9 & $4.72 \pm 1.32$ & 5.3 & $0.0(1)$ & 0.97 \\
$07 / 2006$ & 53938 & 53940 & 4.4 & 397 & 2927 & 0.0901 & 133 & 7.3 & $4.33 \pm 0.70$ & 4.8 & $1.3(2)$ & 0.52 \\
$08 / 2006$ & 53967 & 53977 & 8.4 & 500 & 4261 & 0.0913 & 111 & 5.1 & $2.74 \pm 0.57$ & 3.1 & $15.1(7)$ & 0.035 \\
$09 / 2006$ & 53995 & 54002 & 7.4 & 405 & 4116 & 0.0920 & 26 & 1.3 & $1.31 \pm 0.58$ & 1.5 & $6.0(5)$ & 0.30 \\
$06 / 2007$ & 54264 & 54270 & 5.3 & 333 & 3006 & 0.0924 & 55 & 3.1 & $1.02 \pm 0.60$ & 1.1 & $1.9(6)$ & 0.93 \\
$07 / 2007$ & 54291 & 54304 & 4.4 & 309 & 2412 & 0.0964 & 76 & 4.5 & $2.51 \pm 0.70$ & 2.8 & $15.2(8)$ & 0.056 \\
$08 / 2007$ & 54321 & 54329 & 1.8 & 93 & 849 & 0.0977 & 10 & 1.0 & $0.52 \pm 0.92$ & 0.6 & $2.9(3)$ & 0.41 \\
$09 / 2007$ & 54345 & 54345 & 0.5 & 11 & 114 & 0.1000 & 0 & -0.1 & $<6.60$ & $<7.4$ & - & - \\
2004 & 53171 & 53292 & 24.2 & 1694 & 15098 & 0.0917 & 310 & 7.7 & $2.37 \pm 0.33$ & 2.6 & $2.0(3)$ & 0.57 \\
2005 & 53582 & 53646 & 32.6 & 1931 & 15785 & 0.0930 & 462 & 11.0 & $3.09 \pm 0.35$ & 3.5 & $0.6(2)$ & 0.73 \\
2006 & 53908 & 54002 & 21.5 & 1429 & 12128 & 0.0914 & 320 & 8.8 & $2.84 \pm 0.34$ & 3.2 & $13.5(3)$ & 0.0037 \\
2007 & 54264 & 54345 & 12.0 & 746 & 6381 & 0.0947 & 141 & 5.3 & $1.50 \pm 0.40$ & 1.7 & $3.8(3)$ & 0.28 \\
Total & 53171 & 54345 & 90.3 & 5800 & 49392 & 0.0924 & 1233 & 16.7 & $2.57 \pm 0.18$ & 2.9 & $10.2(3)$ & 0.016 \\
\hline
\end{tabular}

Notes. ${ }^{(a)}$ The integral flux above $400 \mathrm{GeV}$ is calculated using the excess of events above an energy threshold, which differs slightly from the observed excess. The quoted error is statistical only and the $20 \%$ systematic error on the observed flux is not shown. ${ }^{(b)}$ The percentage is calculated relative to the HESS Crab Nebula flux above $400 \mathrm{GeV}$ (Aharonian et al. 2006a). ${ }^{(c)}$ The $\chi^{2}$, degrees of freedom (NDF), and corresponding $\chi^{2}$ probability $P\left(\chi^{2}\right)$ are given for fits of a constant to $I(>400 \mathrm{GeV})$ binned nightly within a dark period, or monthly within a year, or yearly within the total. ${ }^{(d)}$ The upper limit is calculated at a $99.9 \%$ confidence level (Feldman \& Cousins 1998).

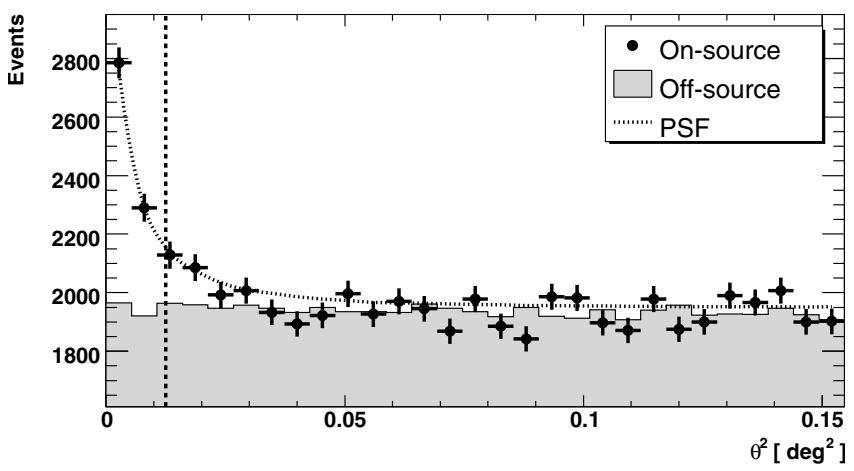

Fig. 1. Distribution of $\theta^{2}$ for on-source events (points with statistical errors) and normalized off-source events (shaded) from observations of PKS 2005-489. The curve represents the $\theta^{2}$ distribution expected from simulations of a $\gamma$-ray point source (photon index $\Gamma=3.20$ ) at a zenith angle of $40^{\circ}$. The dashed line represents the cut on $\theta^{2}$ applied to the data.

of the blazar. The results of the HESS observations are given in Table 1, which shows the dead time corrected observation time, the number of on and off-source events, the on/off normalization $(\alpha)$, the excess and the corresponding statistical significance, for various temporal breakdowns of the HESS data sample.

Figure 1 shows the on-source and normalized off-source distributions of the square of the angular difference between the reconstructed shower position and the source position $\left(\theta^{2}\right)$ for all observations. As can be seen in the figure, the distribution of the excess (i.e. the observed signal) is very similar to what is expected from a simulated point-source of VHE $\gamma$-rays at comparable zenith angles. The off-source distribution is approximately flat in $\theta^{2}$, as expected.
The map of excess counts is well-fit by a two-dimensional Gaussian with a centroid located at $\alpha_{\mathrm{J} 2000}=20^{\mathrm{h}} 09^{\mathrm{m}} 27.0^{\mathrm{s}} \pm$ $1.5_{\text {stat }}^{\mathrm{s}} \pm 1.3_{\text {syst }}^{\mathrm{s}}$ and $\delta_{\mathrm{J} 2000}=-48^{\circ} 49^{\prime} 52^{\prime \prime} \pm 16_{\text {stat }}^{\prime \prime} \pm 20_{\text {syst }}^{\prime \prime}$. As expected, the fit location of HESS J2009-488 is consistent ${ }^{2}$ with the position $\left(\alpha_{\mathrm{J} 2000}=20^{\mathrm{h}} 9^{\mathrm{m}} 25.4^{\mathrm{s}}, \delta_{\mathrm{J} 2000}=-48^{\circ} 49^{\prime} 54^{\prime \prime}\right)$ of the blazar (Johnston et al. 1995). The upper limit (99\% confidence level) on the extension of HESS J2009-488 is $1.0^{\prime}$.

\subsection{VHE flux}

The observed integral flux above $400 \mathrm{GeV}$ for the entire data set is $I(>400 \mathrm{GeV})=\left(2.57 \pm 0.18_{\text {stat }} \pm 0.51_{\text {syst }}\right) \times 10^{-12} \mathrm{~cm}^{-2} \mathrm{~s}^{-1}$. This corresponds to $\sim 2.9 \%$ of the flux above $400 \mathrm{GeV}$ from the Crab Nebula, as determined by HESS (Aharonian et al. 2006a). Figures 2 and 3 show the flux measured for each dark period and night, respectively.

The integral flux $I(>400 \mathrm{GeV})$ observed during various epochs is reported in Table 1 , together with the $\chi^{2}$ and corresponding probability, $P\left(\chi^{2}\right)$, for fits of a constant to the data when binned by nights within each dark period, by dark periods within a year, and by year within the total observations. There are weak indications $\left(P\left(\chi^{2}\right)<0.05\right)$ of variability on annual time scales, monthly time scales in 2006 , and nightly time scales in the dark periods of September 2005 and August 2006. The combined variations on different time scales lead to an indication of variability in the overall monthly curve $\left(\chi^{2}=30.5\right.$ for $13 \mathrm{NDF}$, with $P\left(\chi^{2}\right) \lesssim 0.004$, Fig. 2). Although no variability is seen inside the other epochs (e.g. monthly time scales in 2004, 2005, or 2007), variations in amplitude comparable to the statistical errors cannot be ruled out.

2 The difference between the two positions is $25^{\prime \prime} \pm 28^{\prime \prime}$. 


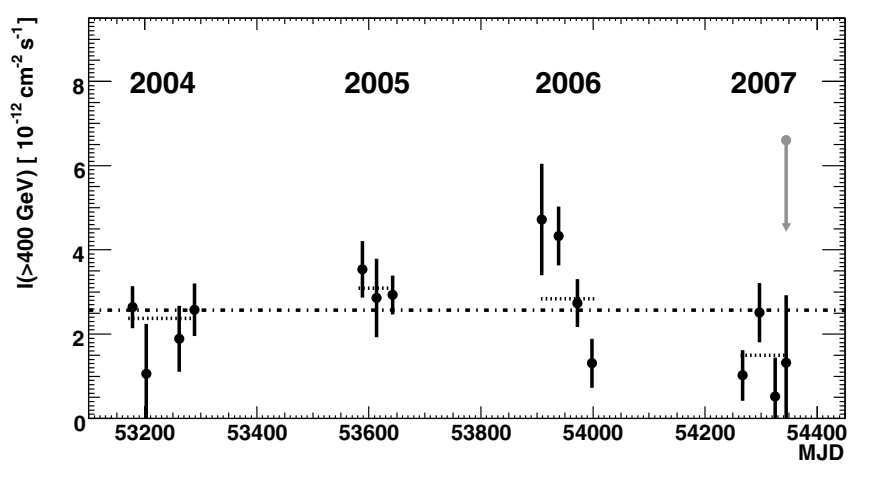

Fig. 2. Integral flux, $I(>400 \mathrm{GeV})$, measured by HESS from PKS 2005-489 during each dark period of observations (i.e. moonless night time within a month). Only the statistical errors are shown. The horizontal line represents the average flux for all the HESS observations. The four horizontal line segments represent the average annual flux observed during the corresponding years (see Table 1).

\subsection{VHE spectra}

The time-average photon spectrum for the entire data set is shown in Fig. 4. All points in the energy range $300 \mathrm{GeV}$ to $\sim 5.3 \mathrm{TeV}$ are significant except the last $(1.5 \sigma)$ at $\sim 4.6 \mathrm{TeV}$. The data are well-fit, $\chi^{2}$ of 9.9 for 8 degrees of freedom, by a power law $\left(\mathrm{d} N / \mathrm{d} E=I_{400}(E / 400 \mathrm{GeV})^{-\Gamma}\right)$ with a photon in$\operatorname{dex} \Gamma=3.20 \pm 0.16_{\text {stat }} \pm 0.10_{\text {syst }}$. Removing the $\sim 4.6 \mathrm{TeV}$ point does not significantly affect the fit result. No evidence is found for significant features, such as a cut-off or break, in the energy spectrum, also considering the upper limits at higher energies.

The time-average spectra measured during each year of datataking are shown in Fig. 5. The results of the best $\chi^{2}$ fits of a power law to these data are shown in Table 2. All spectra are generated with standard cuts (Benbow 2005), except for 2007. Given the short exposure, low flux, steep spectral slope, and degradation of the optical efficiency, a spectrum for 2007 could only be generated with the spectrum cuts (Aharonian et al. 2006b), which lower the energy threshold of the analysis at the expense of sensitivity at higher energies. In Table 2, the epoch, lower and upper energy bounds, photon index, differential flux normalization at $400 \mathrm{GeV}\left(I_{400}\right), \chi^{2}$, degrees of freedom (NDF), and $\chi^{2}$ probability $P\left(\chi^{2}\right)$ for each fit are given. The $\chi^{2}$ probability for a fit of a constant to the annual $\Gamma$ values is 0.30 .

\subsection{Correction to 2004 HESS data}

The data previously published (Aharonian et al. 2005) for HESS observations of PKS 2005-489 in 2004 were not corrected for a $19 \%$ average decrease in the optical efficiency until 2004. In addition, the early simulations neglected the small gaps between Winston cone apertures, the combined effect being an overall correction of $22 \%$ compared to the initial analysis. Figure 6 illustrates the effect of correcting the energy of individual events for the relative optical efficiency of the system, as described earlier. Comparing (see Table 2) the spectrum for 2004 determined here to the previously published one shows significant differences in the flux normalization $\left(I_{400}\right)$ but not in the photon index $(\Gamma)$. The flux measured in 2004 is three times higher than previously published ${ }^{3}$, because of the steep spectrum of the source. It should be noted that, according to the model for the decrease in the optical

\footnotetext{
3 The flux was reported above a threshold of $200 \mathrm{GeV}$. Extrapolating the earlier result, using $\Gamma=4.0$ as reported, yields $I(>400 \mathrm{GeV})=$ $\left(0.86 \pm 0.12_{\text {stat }} \pm 0.17_{\text {syst }}\right) \times 10^{-12} \mathrm{~cm}^{-2} \mathrm{~s}^{-1}$.
}

efficiency of the HESS system, the simulated values match the actual values measured in 2003 , therefore the upper limit published for 2003 HESS observations is unchanged. Extrapolating the 2003 upper limit (99\% confidence level, Aharonian et al. 2005 ) to above $400 \mathrm{GeV}$ (using $\Gamma=4.0$ from the original publication $)$ yields $I(>400 \mathrm{GeV})<\left(0.65 \pm 0.13_{\text {syst }}\right) \times 10^{-12} \mathrm{~cm}^{-2} \mathrm{~s}^{-1}$.

\section{Coordinated XMM-Newton/RXTE observations}

PKS 2005-489 was observed three times with XMM-Newton between 2004 and 2005 (Obs-Id 0205920401 , 0304080301 and 0304080401 ). The satellite pointings were scheduled such that simultaneous HESS observations were possible. The first XMM-Newton pointing was performed on October 4, 2004, and yielded a net exposure of $11 \mathrm{ks}$. Unfortunately poor weather did not allow HESS observations on this night. Good quality HESS data were taken on the following nights. Two more pre-planned XMM-Newton observations were performed on the nights of September 26 and 28, 2005, with exposures of 21 and $25 \mathrm{ks}$, respectively. The goal of scheduling such temporally close ${ }^{4} X M M-N e w t o n$ pointings was to sample both the shortest variability time scales and spectral variations occurring on the typical time scale of HBL (one to a few days; see e.g. Tanihata et al. 2001). It should also be noted that the simultaneous observations had to occur near the end of the HESS observing season for PKS 2005-489, because of the narrow overlap between the HESS and XMM-Newton visibility windows caused by the constraints on the orientation of the solar panels of the satellite.

During the XMM-Newton observations, all three EPIC CCD cameras were used along with the thin filter. The PN detector (Strüder et al. 2001) was operated in timing mode, i.e. when the data from a predefined area of the CCD chip are collapsed into a one-dimensional row to be read out at high speed. This mode allows the sampling of the shortest possible flux and spectral variations without potential pile-up ${ }^{5}$ problems during bright states. The two MOS (Turner et al. 2001) instruments were used in different configurations. MOS1 took data in timing mode in 2004 and in small-window mode during both 2005 observations. The MOS2 camera was operated in small-window mode in 2004 and in large-window mode in 2005. Simultaneous observations were also performed with the Optical Monitor (OM) onboard XMM-Newton, using all photometric filters sequentially. For these data the central window was read in fast mode to enable temporal studies within the data. The exposure for each filter varied between 1800 and $4400 \mathrm{~s}$, ultimately constrained by the overall duration of the pointing.

In August 2005, target-of-opportunity observations of PKS 2005-489 with RXTE were triggered based on apparent enhanced VHE activity in preliminary analysis of uncalibrated HESS data. The satellite pointings were scheduled such that further HESS data could be taken simultaneously. Unfortunately, only a limited subset of the HESS data in this epoch passes standard quality selection criteria because of poor weather conditions in Namibia.

\subsection{XMM-Newton data analysis}

The EPIC data were processed and analyzed with SAS v7.1.0, using the calibration files as of July 2008 and standard screening

\footnotetext{
4 The blazar was observed in 2 consecutive orbits.

5 That is, when more than one X-ray photon arrives in one camera pixel or adjacent pixels before the CCD is read out.
} 
The HESS Collaboration: VHE monitoring of PKS 2005-489 and simultaneous multi-wavelength observations
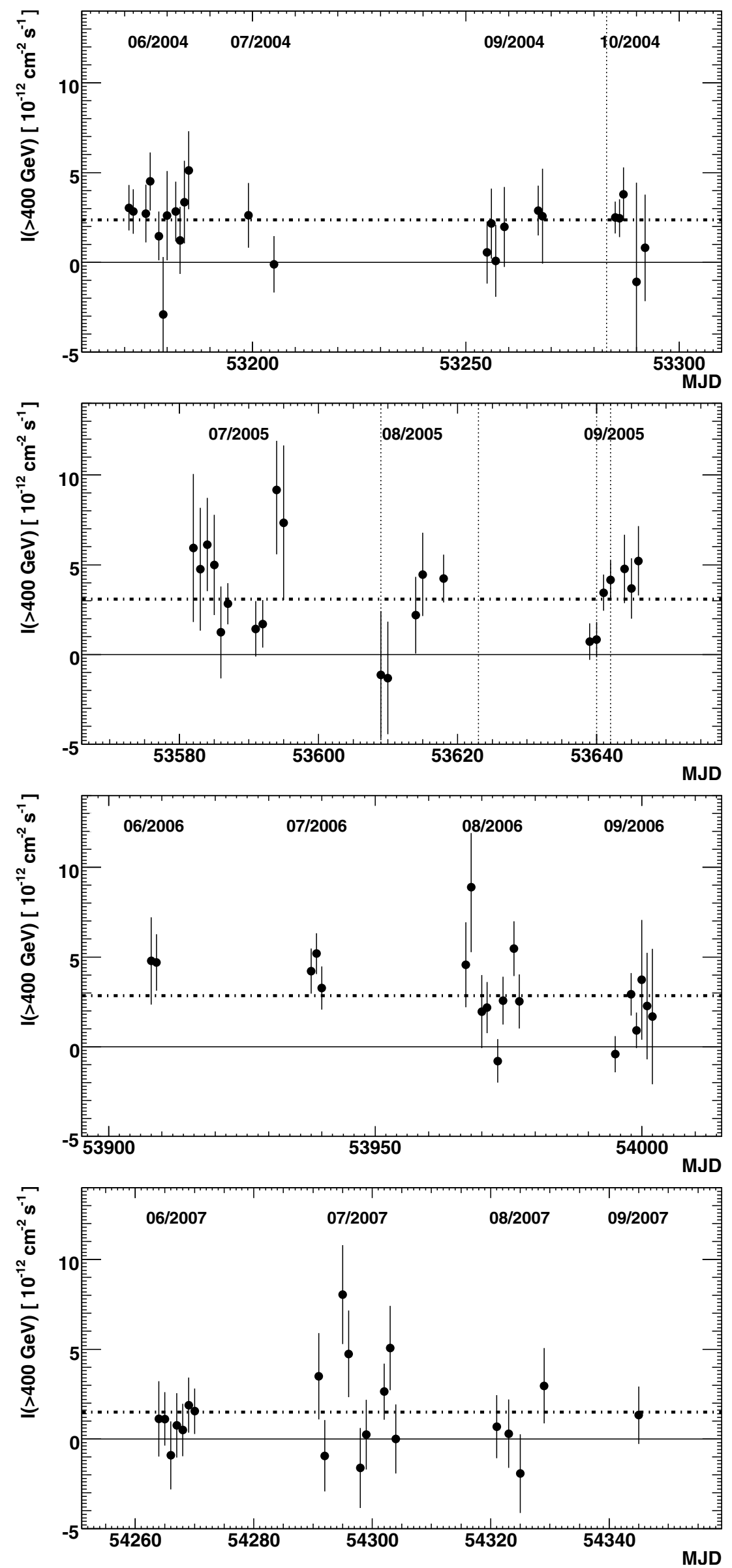

Fig. 3. Integral flux, $I(>400 \mathrm{GeV})$, measured by HESS from PKS 2005-489 during each night of observations. The individual plots represent each of the four years (2004-2007) of data taking. Only the statistical errors are shown. The horizontal lines represent the average annual flux observed during the respective year. The vertical lines at MJD 53282, 53640, and 53642 represent the nights of XMM-Newton observations. The epoch of the RXTE observations is between the vertical lines at MJD 53609 and 53623. 
A\&A 511, A52 (2010)

Table 2. Best $\chi^{2}$ fits of a power-law to the various spectra of PKS 2005-489 measured by HESS.

\begin{tabular}{|c|c|c|c|c|c|c|c|}
\hline Epoch & $\begin{array}{l}E_{\text {lower }} \\
{[\mathrm{TeV}]}\end{array}$ & $\begin{array}{l}E_{\text {upper }} \\
{[\mathrm{TeV}]}\end{array}$ & $\Gamma$ & $\begin{array}{c}I_{400} \\
{\left[10^{-11} \mathrm{~cm}^{-2} \mathrm{~s}^{-1} \mathrm{TeV}^{-1}\right]}\end{array}$ & $\chi^{2}$ & $\mathrm{NDF}$ & $P\left(\chi^{2}\right)$ \\
\hline $2004(\mathrm{AH} 05)^{a}$ & 0.2 & 2.7 & $3.98 \pm 0.38_{\text {stat }} \pm 0.10_{\text {syst }}$ & $0.74 \pm 0.27_{\text {stat }} \pm 0.15_{\text {syst }}$ & 5.6 & 7 & 0.59 \\
\hline $\begin{array}{r}2004^{b} \\
2005 \\
2006 \\
2007^{c}\end{array}$ & $\begin{array}{l}0.30 \\
0.32 \\
0.35 \\
0.24\end{array}$ & $\begin{array}{c}4.0 \\
5.1 \\
11 \\
0.75\end{array}$ & $\begin{array}{l}3.65 \pm 0.39_{\text {stat }} \pm 0.10_{\text {syst }} \\
3.09 \pm 0.22_{\text {stat }} \pm 0.10_{\text {syst }} \\
2.86 \pm 0.20_{\text {stat }} \pm 0.10_{\text {syst }} \\
3.48 \pm 0.68_{\text {stat }} \pm 0.10_{\text {syst }}\end{array}$ & $\begin{array}{l}1.62 \pm 0.33_{\text {stat }} \pm 0.32_{\text {syst }} \\
1.46 \pm 0.28_{\text {stat }} \pm 0.29_{\text {syst }} \\
1.28 \pm 0.20_{\text {stat }} \pm 0.26_{\text {syst }} \\
1.00 \pm 0.22_{\text {stat }} \pm 0.20_{\text {syst }}\end{array}$ & $\begin{array}{c}6.7 \\
13.3 \\
3.7 \\
0.6\end{array}$ & $\begin{array}{l}7 \\
6 \\
7 \\
2\end{array}$ & $\begin{array}{c}0.46 \\
0.038 \\
0.53 \\
0.74\end{array}$ \\
\hline Total & 0.3 & 5.3 & $3.20 \pm 0.16_{\text {stat }} \pm 0.10_{\text {syst }}$ & $1.37 \pm 0.17_{\text {stat }} \pm 0.27_{\text {syst }}$ & 9.9 & 8 & 0.27 \\
\hline
\end{tabular}

Notes. ${ }^{(a)}$ This (AH05) is the previously published HESS result (Aharonian et al. 2005) for 2004, and was not corrected for long-term changes in the optical efficiency of the system. ${ }^{(b)}$ The 2004 entry is the very same data as presented in AH05, but with a correction (see text) applied to account for relative changes in the optical throughput of the HESS array. The 2005, 2006, 2007, and total entries also have this correction applied. (c) The 2007 spectrum is generated with the spectrum cuts (Aharonian et al. 2006b), see Sect. 3.2

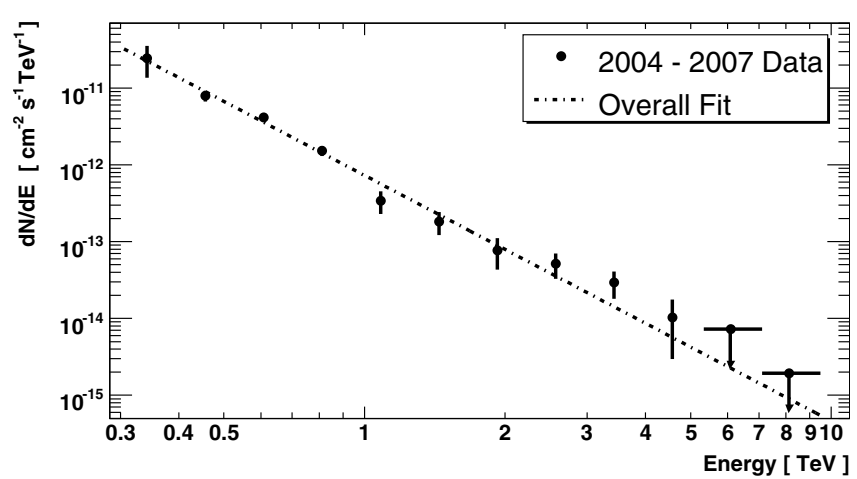

Fig. 4. Time-average VHE energy spectrum observed from PKS 2005-489. The dashed line represents the best $\chi^{2}$ fit of a power law to the observed data (see Table 2), and then extrapolated to $10 \mathrm{TeV}$. Only the statistical errors are shown and the upper limits are at the $99 \%$ confidence level (Feldman \& Cousins 1998).

criteria $^{6}$. A period of $\sim 30$ min at the end of Obs-ID 0304080401 was excluded from the analysis because of high background rates. The MOS1 and MOS2 imaging observations are slightly to moderately affected by pile-up. These effects were explored using epatplot (which computes the fractions of single and double pixel events showing if they differ from normal values), and are sufficiently suppressed by the use of only single-pixel $($ PATTERN $=0)$ events in the analysis. Background events for MOS2 are extracted from an annulus around the source with inner radius $125^{\prime \prime}$ and outer radius $160^{\prime \prime}$. For MOS1 the background information was taken from the same source region in blank-sky observations, since there is no region free of source photons on the same CCD. A check was also performed using background regions from the outer CCDs, as suggested in the SAS Analysis Guide. For the PN, the spectra were extracted from a rectangular box 20 pixels wide, centered on the source strip and extended along the CCD (in raw pixel coordinates, $28 \leq$ RAWX $\leq 48$ ). The background events were extracted from rows $2 \leq$ RAWX $\leq 18$, avoiding the noisier strip on the CCD border.

The spectral analysis was performed with XSPEC v11.3.2ag, testing different binning schemes with at least 50 counts in each new bin. For the 2004 observation, the spectrum from the MOS1 instrument is not included since the timing mode data are significantly noisier than, but consistent with, the other detectors. The MOS and PN spectra are fit together, with

\footnotetext{
${ }^{6}$ see SAS User Guide and CAL-TN-0018.
}

a free constant to allow for different MOS/PN normalizations. The PN flux is adopted as the reference value, but the MOS fluxes are typically within a few percent of the PN fluxes. The $\mathrm{X}$-ray spectra are each fit with single and broken power-law models, with interstellar absorption modelled by TBabs (Wilms et al. 2000). This model is used with the solar abundances of Wilms et al. (2000) and the cross-sections by Verner et al. (1996) (for a discussion, see Baumgartner \& Mushotzky 2006). The results of the fits are shown in Fig. 7 and Table 3 (details in Sect. 5.2).

The data from the OM were processed with the tasks omichain for the photometry and omfchain for the timing analysis. Since no variations are found in the light curves, the whole exposure is used to derive the flux measurements. The photometry was processed interactively with omsource for every filter, to assure the use of point-source analysis procedures. A standard aperture of 6 pixels on the $2 \times 2$ binned images ( 12 for the unbinned images of the 2004 October data set) is used for all filters. This corresponds to an aperture of $6^{\prime \prime}$ for the optical filters $(V$, $B, U)$. The counts for the $U V$ filters are extrapolated by the software, using the UV point-spread function, to an aperture of $177^{\prime \prime} 5$. These are the two apertures for which the OM count-rate-to-flux conversion is calibrated. The OM images are affected by stray light from a bright star in the field of view, which increases the background near one side of the source region. Therefore background events are taken from both an annulus around the source and from two different circular regions at the same distance of the source from the main stray-light reflex. Tests performed with different background sizes and locations show that the photometry does not change by more than $1 \%$, well below the systematic uncertainties of the flux conversion (estimated at $\sim 10 \%$, see XMM-SOC-CAL-TN-0019).

The source fluxes were obtained from the count rates using the OM conversion factors for white dwarfs ${ }^{7}$, adding the $10 \%$ systematic error in quadrature. The fluxes were de-reddened for Galactic absorption using the extinction curve by Cardelli et al. (1989) with the updates by O'Donnell (1994), and assuming $R_{V}\left[=A_{V} / E(B-V)\right]=3.1$. This is the average value for the Galactic diffuse ISM. For the line of sight of PKS 2005-489, a value of $A_{\mathrm{B}}=0.241$ is used (from NED; Schlegel et al. 1998), corresponding to $A_{V(5500)}=0.182$. The conversion factors, extinction ratios and the resulting source fluxes are reported for each of the filters in Table 4.

PKS 2005-489 is hosted by a giant elliptical galaxy of total $R$ magnitude 14.5 and half-light radius 5.7" (from HST snapshot

\footnotetext{
7 http://xmm2.esac.esa.int/sas/7.1.0/watchout/
} 
The HESS Collaboration: VHE monitoring of PKS 2005-489 and simultaneous multi-wavelength observations

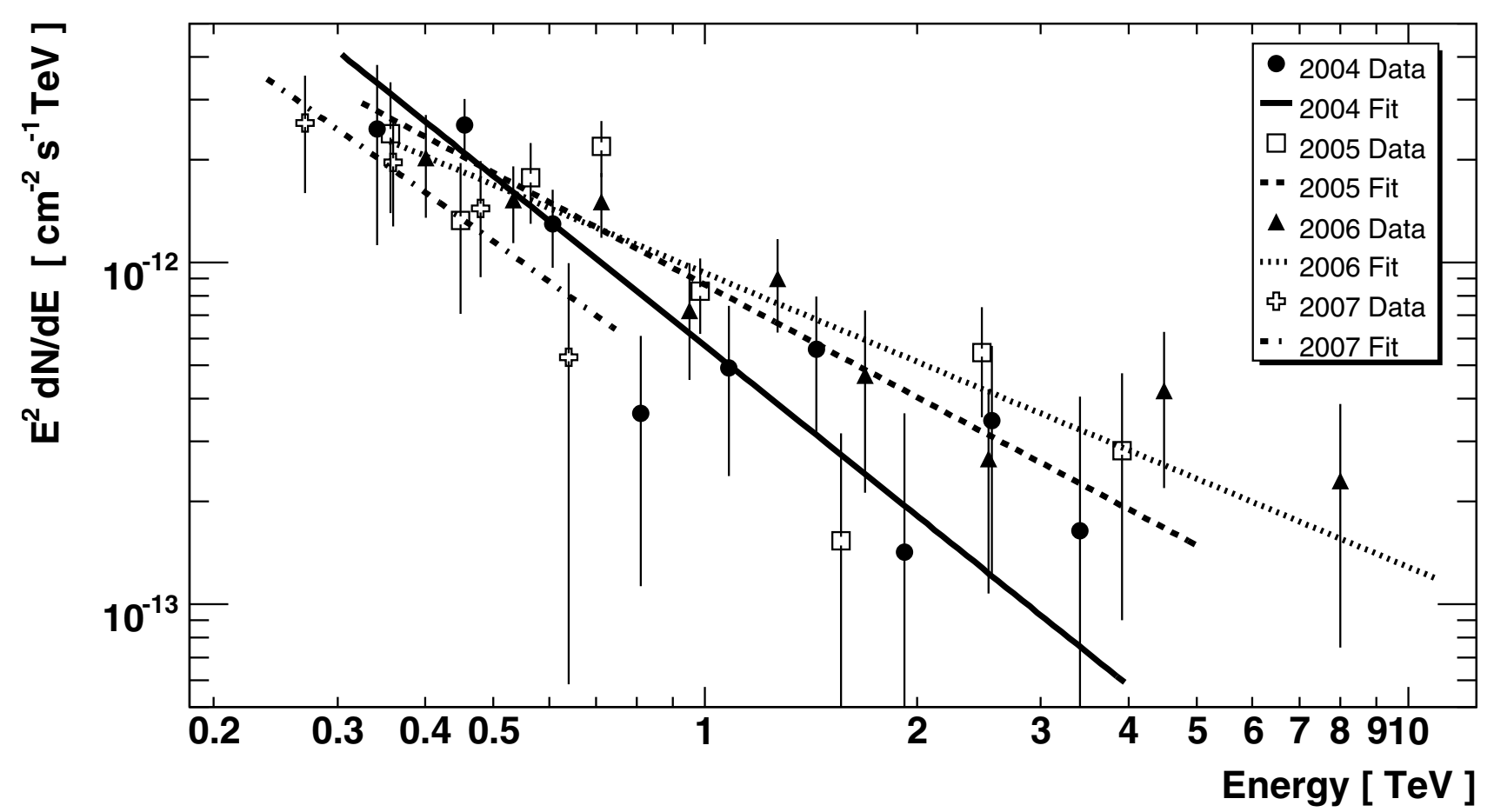

Fig. 5. Photon spectra observed by HESS from PKS 2005-489 during each year of data taking. Only the statistical errors are shown. Each line represents the best $\chi^{2}$ fit of a power law to the observed data.

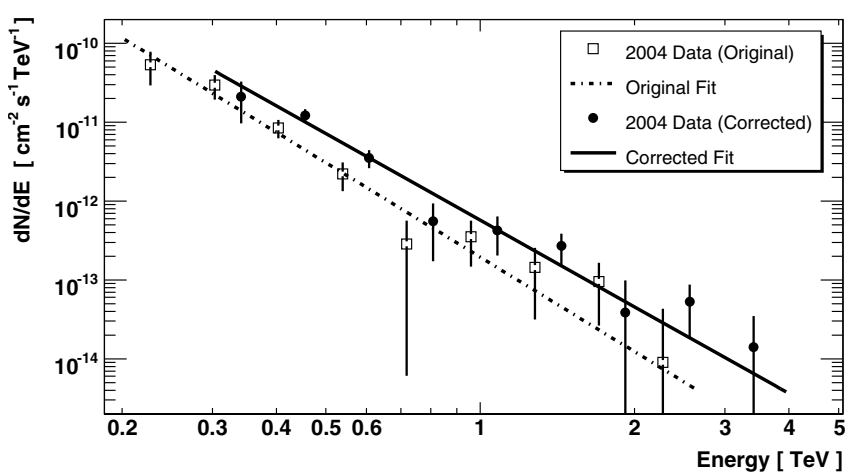

Fig. 6. VHE spectrum measured by HESS from PKS 2005-489 in 2004 compared to the previously published version (Aharonian et al. 2005). Only the statistical errors are shown. Correcting the 2004 data for decreases in the optical efficiency of the HESS array results in a threetimes higher integral flux above $400 \mathrm{GeV}$.

observations, Scarpa et al. 2000). For the SEDs (Figs. 9 and 10), the OM fluxes were corrected for the contribution of the host galaxy. The wavelength-dependent correction was determined using a template SED for elliptical galaxies (Silva et al. 1998), rescaled to the host-galaxy flux in the $R$ band, and accounts for the given apertures. The OM fluxes are always dominated by the non-thermal emission, and the small contribution of the hostgalaxy is only noticeable in the $V$ and $B$ filters.

\subsection{RXTE data analysis}

The RossiXTE/PCA (Jahoda et al. 1996) performed 15 snapshot observations of PKS 2005-489 between August 20 and September 19, 2005, yielding a total exposure of $23.4 \mathrm{ks}$. The observations were mostly done with PCU0 and PCU2. The PCA STANDARD2 data were reduced and analyzed with the standard FTOOLS routines in HEASOFT V6.3.2, using the filtering criteria recommended by the RXTE Guest Observer Facility. The new SAA history file and parameters for the background calculation provided in September 2007 were used, which correct a bug in the calculation of the faint background model ${ }^{8}$. Only the top-layer events were processed and only the PCU2 data were considered, to ensure a more accurate spectral measurement. The average net count rate in the $3-15 \mathrm{keV}$ band is $0.54 \pm 0.02 \mathrm{cts} \mathrm{s}^{-1} \mathrm{pcu}^{-1}$. The RXTE spectra were extracted and fitted separately for each pointing, and summed together to obtain the average spectrum. Each spectrum is well-fit by a single power-law model.

\section{XMM-Newton/RXTE results}

\subsection{Flux variability}

The 2004 and both 2005 EPIC light curves contain no evidence of flux variability within each exposure, and have average net count rates in the MOS2 camera of $1.14 \pm 0.04,6.38 \pm 0.08$, and $6.15 \pm 0.08$ counts $s^{-1}$, respectively. The probability of constant emission is $P_{\text {const }} \geq 0.99,0.92$ and 0.99 for the three epochs, respectively. Similarly, no flux variability is observed in the OM data for any of the observed filters. No significant flux variations $\left(P_{\text {const }} \geq 0.76\right)$ are detected during the RXTE observations (see Fig. 8) as well. However, night-by-night flux variations of a few tens of percent cannot be excluded, due to low statistics in the RXTE measurements.

Although no variability is found within any of the XMM-Newton exposures or within the complete RXTE sample, strong flux variations are found on longer time scales. The $2-10 \mathrm{keV}$ flux varies by a factor of $\sim 16$ between 2004 and 2005. In the UV and optical bands the flux increases by $\sim 40 \%$ and $\sim 20 \%$, respectively, between 2004 and 2005. A smaller (factor of 2.5), but significant, change in the $2-10 \mathrm{keV}$

\footnotetext{
${ }^{8}$ Details at http://www. universe.nasa.gov/xrays/programs/ rxte/pca/doc/bkg/bkg-2007-saa/
} 

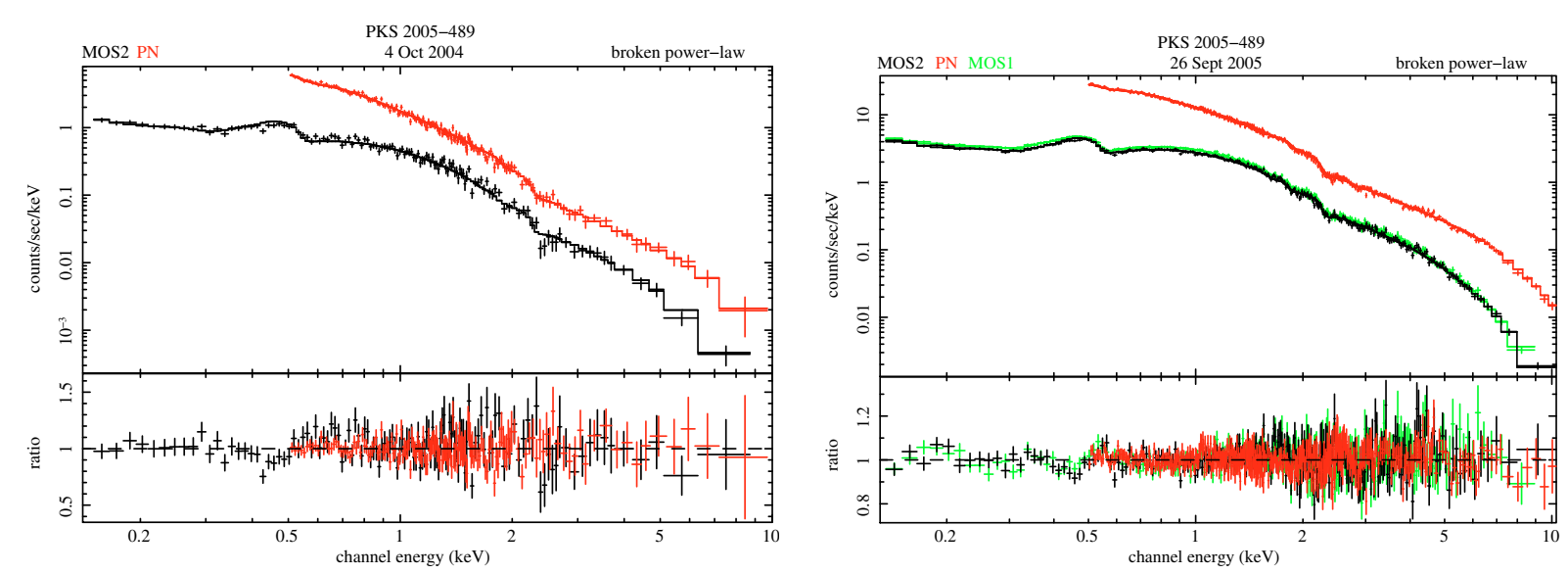

Fig. 7. Best-fit data and folded model, plus residuals, of the XMM-Newton PN (upper) and MOS2/1 (lower) spectra of PKS 2005-489, in October 4, 2004 (left panel) and September 26, 2005 (right panel). The spectra are fitted with a concave broken power-law model plus Galactic absorption.

Table 3. Fits to the X-ray spectra ${ }^{a}$ in the different epochs.

\begin{tabular}{|c|c|c|c|c|c|c|c|c|c|}
\hline Instrument & $\begin{array}{c}\text { Band } \\
\mathrm{keV}\end{array}$ & Date & Model $^{b}$ & $\Gamma_{1}$ & $\begin{array}{c}E_{\text {break }} \\
\mathrm{keV}\end{array}$ & $\Gamma_{2}$ & $\begin{array}{r}F_{0.1-2.0 \mathrm{keV}^{b}} \\
\mathrm{erg} \mathrm{cm}\end{array}$ & $\begin{array}{l}F_{2-10 \mathrm{keV}^{b}} \\
2 \mathrm{~s}^{-1}\end{array}$ & $\chi_{\mathrm{r}}^{2}($ \\
\hline $\mathrm{XM}$ & 0.15 & 4 & $\begin{array}{l}\text { p.l. } \\
\text { b.p.l. }\end{array}$ & $\begin{array}{l}3.04 \pm 0.02 \\
3.06 \pm 0.02\end{array}$ & $\begin{array}{c}- \\
2.5 \pm 0.5\end{array}$ & $\begin{array}{c}- \\
2.64 \pm 0.20\end{array}$ & $\begin{array}{l}2.46 \times 10^{-11} \\
2.77 \times 10^{-11}\end{array}$ & $\begin{array}{l}1.03 \times 10^{-12} \\
1.19 \times 10^{-12}\end{array}$ & $\begin{array}{l}1.110(284) \\
1.048(282)\end{array}$ \\
\hline RXTE PCU2 & $3-20$ & 20/8-19/9/05 & p.l. & - & - & $2.90 \pm 0.18$ & - & $7.54 \times 10^{-12}$ & $0.79(16)$ \\
\hline $\mathrm{XMM}$ Mos+PN & $0.15-10$ & $26 / 9 / 05$ & $\begin{array}{l}\text { p.l. } \\
\text { b.p.l. }\end{array}$ & $\begin{array}{l}2.31 \pm 0.01 \\
2.34 \pm 0.01\end{array}$ & $\begin{array}{c}- \\
0.79 \pm 0.11\end{array}$ & $\begin{array}{c}- \\
2.30 \pm 0.01\end{array}$ & $\begin{array}{l}7.55 \times 10^{-11} \\
7.72 \times 10^{-11}\end{array}$ & $\begin{array}{l}1.93 \times 10^{-11} \\
1.95 \times 10^{-11}\end{array}$ & $\begin{array}{l}1.138(860) \\
1.122(858)\end{array}$ \\
\hline XMN & $0.15-10$ & 28/9/05 & $\begin{array}{l}\text { p.l. } \\
\text { b.p.l. }\end{array}$ & $\begin{array}{l}2.37 \pm 0.01 \\
2.39 \pm 0.01\end{array}$ & $\begin{array}{c}- \\
1.78 \pm 0.15\end{array}$ & $\begin{array}{c}- \\
2.31 \pm 0.02\end{array}$ & $\begin{array}{l}8.01 \times 10^{-11} \\
8.19 \times 10^{-11}\end{array}$ & $\begin{array}{l}1.77 \times 10^{-11} \\
1.83 \times 10^{-11}\end{array}$ & $\begin{array}{l}1.509(888) \\
1.372(886)\end{array}$ \\
\hline
\end{tabular}

Notes. ${ }^{(a)}$ The errors are reported at a $90 \%$ confidence level for 1 parameter $\left(\Delta \chi^{2}=2.71\right)$. ${ }^{(b)}$ Fits with fixed Galactic $N_{\mathrm{H}}=3.93 \times 10^{20} \mathrm{~cm}^{-2}$; power-law (p.l.) and broken power-law (b.p.l.) models. Unabsorbed fluxes.

Table 4. Optical Monitor parameters and source fluxes for the three XMM-Newton observations.

\begin{tabular}{lcccccc}
\hline \hline Filter & $\lambda_{\mathrm{e}}{ }^{a}$ & $A_{\lambda_{\mathrm{e}}} / A_{\mathrm{V}}{ }^{b}$ & $F_{\text {conv }}{ }^{c}$ & $F_{4 / 10}{ }^{d}$ & $F_{26 / 9}{ }^{d}$ & $F_{28 / 9}{ }^{d}$ \\
\hline$V$ & 5430 & 1.016 & $2.49 \times 10^{-16}$ & 11.84 & 14.51 & 14.42 \\
$B$ & 4500 & 1.293 & $1.29 \times 10^{-16}$ & 9.64 & 12.08 & 12.09 \\
$U$ & 3440 & 1.634 & $1.94 \times 10^{-16}$ & 7.48 & 9.79 & 9.78 \\
$U V W 1$ & 2910 & 1.870 & $4.76 \times 10^{-16}$ & 6.12 & 8.27 & 8.14 \\
$U V M 2$ & 2310 & 2.827 & $2.20 \times 10^{-15}$ & 5.57 & 7.74 & 7.71 \\
$U V W 2$ & 2120 & 3.167 & $5.71 \times 10^{-15}$ & - & - & 7.08 \\
\hline
\end{tabular}

Notes. ${ }^{(a)}$ The effective wavelength of the filter in $\AA .{ }^{(b)}$ The ratio of $A_{\lambda_{\mathrm{e}}}$, i.e. the extinction in magnitudes at the effective wavelength of the filter $\lambda_{\mathrm{e}}$, to $A_{V}(5500 \AA$ ) obtained from the interstellar reddening curve given by Cardelli et al. (1989) and updated by O'Donnell (1994). ${ }^{(c)}$ The conversion factor from rate (Counts s $\left.{ }^{-1}\right)$ to flux $\left(\mathrm{erg} \mathrm{cm}^{-2} \mathrm{~s}^{-1} \AA^{-1}\right)$ from the OM in-orbit calibration (see SAS watch-out page). ${ }^{(d)}$ The flux of PKS 2005-489, corrected for galactic extinction, measured in the three epochs (Oct. 4, 2005; Sept. 26 and 28, 2005). The units are mJy.

flux also occurs during the 17 days between the final RXTE and first XMM-Newton pointing in September 2005.

\subsection{X-ray spectrum}

Since there is no variability in the X-ray flux or hardness ratio, during each XMM-Newton exposure, a single X-ray spectrum was extracted for each of the three pointings (see Table 3 and
Fig. 7). The spectra from the individual RXTE exposures do not vary significantly (see Fig. 8) and are consistent with the fit to the complete RXTE sample reported in Table 3. In each case (XMM-Newton and RXTE), the Galactic absorption is fixed to $N_{\mathrm{H}}=3.93 \times 10^{20} \mathrm{~cm}^{-2}$, as recently determined from the LAB survey (Kalberla et al. 2005). It is important to note that there is a discrepancy between the LAB survey estimate and the value obtained from the HI maps by Dickey \& Lockman (1990) (DL, $N_{\mathrm{H}}=5.08 \times 10^{20} \mathrm{~cm}^{-2}$ ). The LAB survey value is adopted because it provides better residuals, is consistent with the results when $N_{\mathrm{H}}$ is left as free parameter $\left((3.96 \pm 0.17) \times 10^{20} \mathrm{~cm}^{-2}\right)$, and is close to the value previously adopted for the BeppoSAX results on the large flare of $1998\left(4.2 \times 10^{20} \mathrm{~cm}^{-2}\right.$, Tagliaferri et al. 2001). Using the DL value significantly changes the slope of the source spectrum below $1 \mathrm{keV}$. Therefore both cases are considered for interpreting the fits results, as additional systematic uncertainty.

In all three data sets, the EPIC spectra are better fit by a broken power-law model with respect to a single power law, with high significance (F-test $>99.9 \%$ ). Remarkably, the parameters reveal an inverted broken power-law spectrum, where the slope in the soft X-ray band is steeper than in the hard X-ray band $\left(\Gamma_{2}<\Gamma_{1}\right)$.

In the 2004 dataset, the X-ray spectrum is characterized by the lowest flux ever recorded from this source (see Fig. 9) and a very steep slope of $\Gamma \simeq 3$ up to $\sim 3 \mathrm{keV}$. At higher energies, there is evidence of a moderate hardening trend. The trend is consistent between the MOS and PN spectra, but is only significant in 
The HESS Collaboration: VHE monitoring of PKS 2005-489 and simultaneous multi-wavelength observations

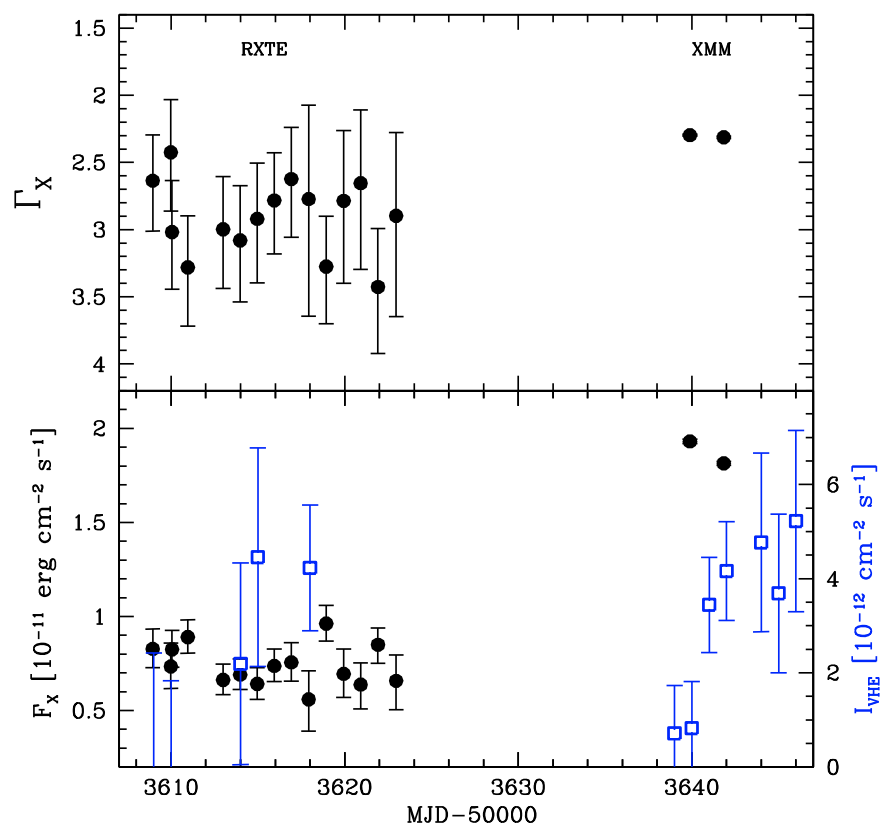

Fig. 8. Simultaneous X-ray and VHE data taken by RXTE, $X M M-N e w t o n$ and HESS in August-October 2005. Upper panel: the $\mathrm{X}$-ray photon index. The XMM-Newton values were obtained from fits above $2 \mathrm{keV}$, to be consistent with the RXTE energy range. Lower panel: the observed X-ray and VHE flux. The X-ray data (filled circles) are energy fluxes integrated in the $2-10 \mathrm{keV}$ band. The HESS data (open squares) are integral photon fluxes above $400 \mathrm{GeV}$. The vertical scales on the left and right are for the X-ray and HESS data, respectively. All errors are shown at the $1 \sigma$ confidence level.

the PN data thanks to better statistics. This hardening can also be reproduced by the sum of two power-law models, the second having a harder index. The slope of this second power-law function is not well constrained. However, assuming a fixed value of $\Gamma=1.5$, as could be expected for the inverse Compton emission of low-energy electrons, a good fit $\left(\chi_{\mathrm{r}}^{2}=1.07\right.$ for $\left.283 \mathrm{NDF}\right)$ is obtained with a flux normalization at $1 \mathrm{keV}$ for the second power-law function at $2.8 \pm 0.7 \%$ of the first power law. In this case, the two power-law functions would cross at about $20 \mathrm{keV}$. Using the high $N_{\mathrm{H}}$ has minimal impact on this result, steepening the low-energy slope by only +0.1 .

The difference between the EPIC spectra of September 26 and 28, 2005 is negligible. However, the spectrum from either of the 2005 observations is significantly harder and brighter than in 2004 (Fig. 9). Either 2005 spectrum is represented well by a pure power-law model with $\Gamma=2.3$ from $\sim 1$ to $10 \mathrm{keV}$. At lower energies, each has a slightly steeper slope. Although modest $(\Delta \Gamma \lesssim 0.1)$, the spectral break is significant (F-test $>99.99 \%)$ and is not caused by the extension of the MOS data at low energies, or by the cross-calibration between the detectors. The broken power-law model is also statistically required for the PN spectrum alone (F-test $>99.99 \%$ ), as well as for the MOS spectra fitted separately. It should be noted however that the precise location of the break is affected by systematic more than statistical errors. In Table 3, the break energy for the fit with the overall minimum $\chi^{2}$ is reported, but there are other similar local minima between 0.7 and $2.2 \mathrm{keV}$, whose hierarchy in $\chi^{2}$ can change according to the calibration of the effective area near the instrumental $\mathrm{Si}$ and $\mathrm{Au}$ edges (at 1.7 and $2.1 \mathrm{keV}$ ). The true break therefore should be considered more realistically inside the range $0.7-2.2 \mathrm{keV}$. This does not affect the values of the soft

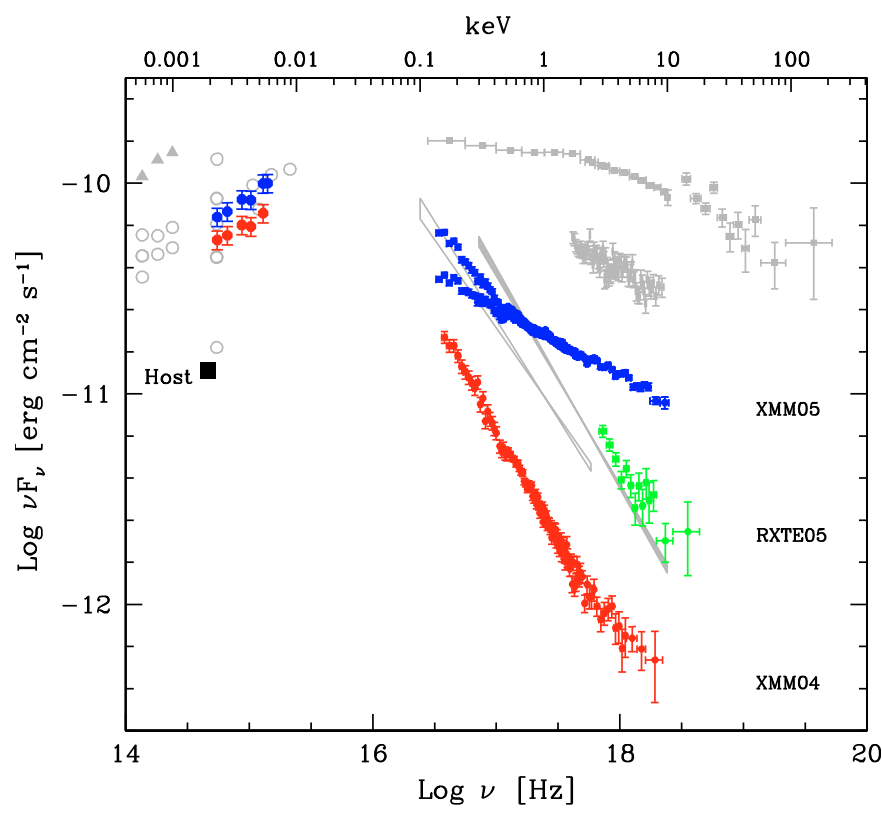

Fig. 9. SED of PKS 2005-489 in the frequency range covered by the XMM-Newton and RXTE observations (filled circles). Archival X-ray spectra are shown along with those measured by XMM-Newton on Oct. 4, 2004 (red points), RXTE in Aug.-Sept. 2005 (green points) and XMM-Newton on Sept. 26, 2005 (blue points). For the latter, two fits are plotted, showing the effects of using two different estimates of the Galactic column density $\left(N_{\mathrm{H}}=5.08\right.$ or $3.93 \times 10^{20} \mathrm{~cm}^{-2}$; see text $)$. For better visibility, the XMM-Newton spectrum of Sept. 28, 2005 is not plotted as it would appear superposed on the Sept. 26 data. The flux of the host galaxy in the $R$ band is plotted as black square. Historical data are shown in gray (from NED and Tagliaferri et al. 2001). In the $\mathrm{X}$-ray range, they correspond to (from top to bottom): the BeppoSAX data of the 1998 flare (Tagliaferri et al. 2001), the BeppoSAX 1996 data (Padovani et al. 2001), the Swift spectrum in April 2005 (filled bow-tie, Massaro et al. 2008), and the 1992 ROSAT spectrum (open bow-tie, Comastri et al. 1997).

and hard slopes in a relevant way (they do not change by more than 0.04 and 0.02 , respectively).

The amount of the spectral break, however, depends on the adopted value of the Galactic column density. Using the DL value instead of the LAB survey value, the soft X-ray slope becomes steeper $\left(\Gamma_{1}=2.6\right)$, making the break more pronounced $(\Delta \Gamma \simeq 0.3)$. The two cases are shown in Fig. 9, where the spectra are plotted together with the other data sets and archival data. Allowing instead the column density to be lower than all available estimates, a single power-law spectrum is obtained with $N_{\mathrm{H}}=3.76 \pm 0.08 \mathrm{~cm}^{-2}\left(\chi_{\mathrm{r}}^{2}=1.226\right.$ for $\left.859 \mathrm{NDF}\right)$, though a broken power-law model still seems to provide a better representation (F-test $>95 \%$ ).

The 2005 RXTE observations yield a very steep $(\Gamma=2.9)$ spectrum from $3-15 \mathrm{keV}$. This spectrum is almost parallel to the 2004 XMM-Newton spectrum but with $\sim 6$ times higher flux. The RXTE photon index and flux are in good agreement with the values measured by Swift in April 2005 (Massaro et al. 2008). This suggests that the hardening of the X-ray spectrum of PKS 2005-489 occurred in September 2005, between the RXTE and XMM-Newton pointings.

\section{Discussion}

The spectral energy distribution (SED) of PKS 2005-489, assembled with the data taken in 2004 and 2005, is shown in 


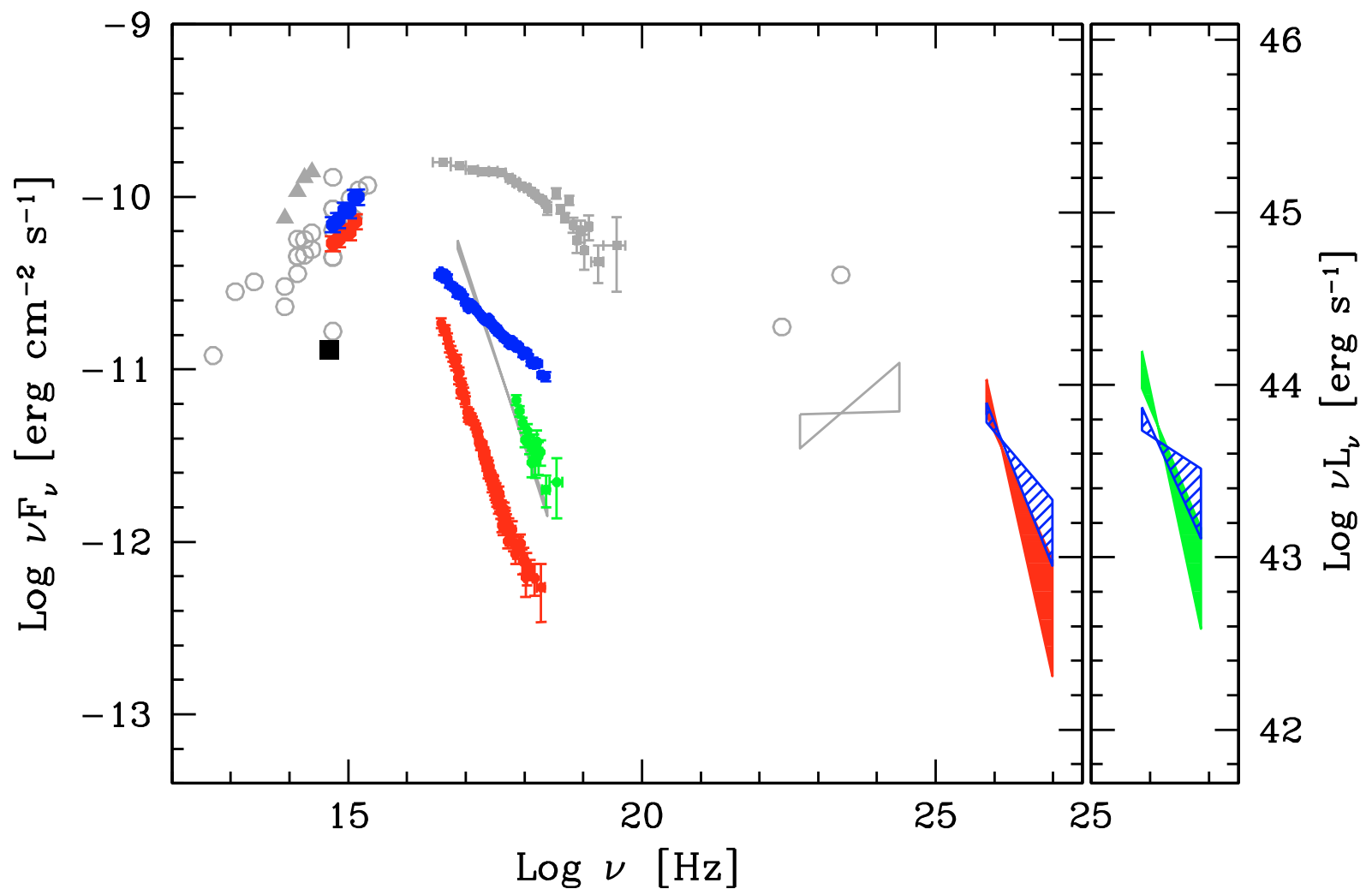

Fig. 10. SED of PKS 2005-489, in different epochs and states. The XMM-Newton and RXTE data are plotted as in Fig. 9, together with historical data (shown in grey; from Tagliaferri et al. 2001; Massaro et al. 2008). The recent Fermi-LAT spectrum $(0.2<E<10 \mathrm{GeV})$ from Aug.-Oct. 2008 is also plotted, for reference (open bow-tie; Abdo et al. 2009). For clarity, the HESS spectra $(E>300 \mathrm{GeV})$ are plotted as bow-ties, and have been corrected for EBL absorption with the model by Franceschini et al. (2008, see Table 5). Main panel: the HESS time-averaged spectrum from 2004 (red filled bow-tie) and 2005 (blue hatched bow-tie). Right mini-panel: the HESS spectrum in 2005, divided in two subsets: before the XMM pointings (periods 07-08/2005 in Fig. 3; green filled bow-tie) and during the XMM epoch (period 09/2005 in Fig. 3; blue hatched bow-tie). Same colors associate optical, X-ray and VHE datasets corresponding to same epochs.

Fig. 10. The VHE $\gamma$-ray spectra are corrected for the energydependent attenuation caused by interactions with the opticalIR photons of the diffuse extragalactic background light (EBL). The correction was performed adopting the EBL model of Franceschini et al. (2008). This model takes into account the most recent results on galaxy emission and evolution, and agrees with both the upper limits on the EBL derived from blazar spectra (Aharonian et al. 2006c, 2007) and the lower limits given by galaxy counts, at both optical and infrared wavelengths (Madau \& Pozzetti 2000; Fazio et al. 2004). The fits to the absorptioncorrected spectra are reported in Table 5, and plotted in Fig. 10.

The simultaneous observations do not show evidence of strong changes in the location of the SED peaks over the years, with respect to the historical values. The hard optical-UV spectrum from the OM photometry and the steep X-ray spectrum above $0.1 \mathrm{keV}$ locate the synchrotron peak between the two bands, at approximately $0.5 \times 10^{16} \mathrm{~Hz}$. At VHE, the steep spectra constrain the peak of the $\gamma$-ray emission to energies $<0.2 \mathrm{TeV}$. The low flux in the VHE band does not enable a determination of the spectral properties in exactly the same (short) observing windows of the X-ray observations. However, given the lack of significant VHE variability in 2004 or 2005, the monthly or yearly average spectra can be considered a reasonable approximation to the VHE spectra during the epoch of the X-ray observations.

In 2004, PKS 2005-489 was in a very low state in both the X-ray and VHE bands. The spectral slopes are also simi$\operatorname{lar}(\Gamma \sim 3)$ for the X-ray and VHE emission. The X-ray flux
Table 5. Best power-law fits to the HESS spectra corrected for EBL absorption following Franceschini et al. (2008).

\begin{tabular}{lccc}
\hline \hline Epoch & $\Gamma_{\text {int }}{ }^{a}$ & $\begin{array}{c}v F_{v}(1 \mathrm{TeV})^{b} \\
{\left[10^{-12} \mathrm{erg} \mathrm{cm}^{-2} \mathrm{~s}^{-1}\right]}\end{array}$ & $\chi^{2}(\mathrm{NDF})$ \\
\hline 2004 & $3.1 \pm 0.4$ & $1.8 \pm 0.4$ & $6.4(7)$ \\
2005 & $2.6 \pm 0.2$ & $2.7 \pm 0.4$ & $13.2(6)$ \\
2006 & $2.4 \pm 0.2$ & $2.9 \pm 0.4$ & $4.3(7)$ \\
2007 & $3.1 \pm 0.7$ & $1.2 \pm 0.9$ & $0.5(2)$ \\
$20057-8^{c}$ & $3.2 \pm 0.4$ & $2.4 \pm 0.6$ & $9.2(4)$ \\
$20059^{c}$ & $2.5 \pm 0.3$ & $2.9 \pm 0.5$ & $4.7(6)$ \\
Total & $2.69 \pm 0.16$ & $2.34 \pm 0.20$ & $8.9(8)$ \\
\hline
\end{tabular}

Notes. ${ }^{(a)}$ Only the statistical errors are shown. ${ }^{(b)}$ The normalization at $1 \mathrm{TeV}$, in $v F_{v}$ units. ${ }^{(c)}$ The HESS spectrum in 2005, extracted in two different epochs (see Fig. 3): before the XMM pointings (observing periods 2005, 7-8) and during the XMM epoch (period 2005, 9).

around few $\mathrm{keV}$ is more than two orders of magnitude less than the BeppoSAX flux during the active state of 1998. The slight spectral hardening towards higher X-ray energies is consistent with the onset of the concavity expected from the emergence of the inverse-Compton emission in the SED. This emission, produced by low-energy electrons, is usually not visible in the X-ray spectra of HBL because of the dominance of the synchrotron emission from high-energy electrons. In this data set, its presence becomes detectable because of the steepness and very low flux of the synchrotron (X-ray) spectrum. A similar behavior has 
recently been observed in the HBL PKS 2155-304 during a low state (Foschini et al. 2008; Zhang 2008). This transition between steep synchrotron and flat inverse-Compton spectra is instead fully visible inside the observed X-ray passband for intermediate BL Lac objects (e.g. ON 231, see Tagliaferri et al. 2000).

In 2005, the X-ray spectrum changes radically. Swift and RXTE measurements made in April and August-September 2005, respectively, show a large increase in flux but little change in the spectral slope compared to 2004. Shortly after the RXTE observations, a much harder spectrum $\left(\Delta \Gamma_{X} \simeq 0.7\right)$ and a brighter state than in the previous epochs is found during $X M M-N e w t o n$ observations. Most interestingly, the data require a spectral break with a steeper spectrum towards lower energies. This break is more apparent when a higher galactic $N_{\mathrm{H}}$ is used (e.g. the DL versus LAB survey values; see Fig. 9), but even when considering a single power-law shape with an ad-hoc $N_{\mathrm{H}}$, the extrapolation of the X-ray spectrum down to lower frequencies does not match the UV-optical data, which are still rising with frequency. A hump with a steeper soft X-ray slope is needed to smoothly connect with the optical-UV spectrum, and this feature indicates the presence of two particle populations, since it cannot be easily reproduced by synchrotron radiation with a single electron distribution.

At VHE, the comparison between the 2004 and 2005 spectra indicates a hardening trend as well, though with marginal significance ( $\sim 1$ sigma; see Table 5$)$ due to the large errors on the HESS measurement. However, if this trend is real, and if the VHE spectrum is indeed produced by the same particles as are responsible for the X-ray emission (as suggested by the similar spectral slopes in 2004-2005 and by the location of the synchrotron peak ${ }^{9}$ ), a similar behavior is expected. Namely, the VHE spectral hardening should have occurred after the RXTE observations, during the epoch of the XMM-Newton pointings, while the 2005 July-August VHE spectrum should be similar to the 2004 spectrum (modulo normalization).

To test this hypothesis, the 2005 HESS data set was divided into two subsets. The first consists of all VHE data before the XMM-Newton pointings (2005 July-August dark periods), and the second contains all 2005 HESS data taken during or after the pointings (2005 September dark period). Table 5 shows the results of power-law fits to the spectra from these subsets, after correction for the absorption of VHE photons on the EBL. The HESS spectrum in the XMM epoch is indeed harder $\left(\Delta \Gamma_{\text {int }}=0.72 \pm 0.47\right)$ than the pre-XMM spectrum, and the statistical significance of the spectral change increases $(\sim 2 \sigma)$ with respect to the comparison between yearly spectra. In addition, the pre-XMM spectrum has the same slope as the 2004 HESS spectrum. The HESS data therefore are fully consistent with a behavior in the VHE band that mirrors the spectral variations seen in the X-ray band, as expected if both emissions are produced by the same electrons. Interestingly, the harder VHE state continues in 2006. Because of the limited exposure in 2007, the VHE statistics are too poor to draw any meaningful conclusions.

The most remarkable feature of the VHE emission, however, is the lack of flux variability. Comparing the average states in 2004 and 2005, the integrated energy flux (e.g. for reference in the decade $0.3-3 \mathrm{TeV}$ ) does not vary by more than $40 \%$ between 2004 and 2005, and is consistent with a constant flux.

\footnotetext{
9 If the VHE electrons were emitting by synchrotron at energies well below the X-ray range, they would correspond to the synchrotron peak, around $10^{16} \mathrm{~Hz}$. Since the SSC scattering at the peak would be in the Thomson regime for this frequency, the gamma-ray peak should then be inside the VHE band, which is not observed.
}

In contrast, the $\mathrm{X}$-ray flux increased by a factor of $\sim 16$ in the $2-10 \mathrm{keV}$ band (see Table 3 ). The optical-UV emission, which is close to the synchrotron peak and which typically provides the target photons for the IC scattering in the VHE band, shows an increase as well ( $40 \%)$. In a homogeneous SSC scenario, if the VHE emission is produced by the same electrons emitting in the $\mathrm{X}$-ray band (as indicated in this case by the spectral behavior), a fresh injection of electrons should make the VHE flux increase at least linearly with the X-ray flux. The reason is that the energy density of all possible seed photons for the IC scattering has increased or is constant between the two epochs. For the VHE flux to remain constant, a corresponding decrease in the seed photons energy density is required.

The X-ray and VHE data taken together, therefore, suggest that a new jet component is emerging in the SED of PKS 2005489 , which is physically separated from the main emitting region. The emission at the synchrotron peak of this new component should be lower, remaining hidden below the observed SED, while its harder spectrum at high energies emerges in the hard-X band. The electrons of this new component would not see the energy density of the observed synchrotron peak, but the lower energy density of their self-produced synchrotron peak instead.

\section{Conclusions}

PKS 2005-489 is detected at VHE in each of the four years it was being observed by HESS (2004-2007). The 2005-2007 data clearly confirm the VHE discovery reported by HESS (Aharonian et al. 2005) and quadruple the statistics of the initial spectrum measurement. Re-analysis of the previously published 2004 HESS data, using the improved calibration of the detector's energy scale, results in a $\sim 3$ times higher flux and a similar photon index.

The measured VHE $\gamma$-ray flux is low $(\sim 3 \% \mathrm{Crab})$ and only shows weak variations on time scales ranging from days to years. The observed time-averaged (2004-2007) VHE spectrum is soft, with a photon index $\Gamma=3.20 \pm 0.16$. Although evidence of VHE spectral variations is marginal by itself, the VHE spectrum seems to track the X-ray slope variations when multiwavelength coverage is available. Observations performed with XMM-Newton and RXTE in 2004 and 2005 reveal remarkable changes in the X-ray spectrum, but without shifting the location of the synchrotron peak with respect to historical observations.

Interpreting these measurements along with the HESS data suggests the emergence of a new jet component in the SED that is characterized by a harder electron spectrum. This component must be separate: its particles cannot interact with the synchrotron photons of the observed SED peak, otherwise higher VHE fluxes than observed would be implied.

PKS 2005-489 is found overall in a very low state, in both the X-ray and VHE bands, during the observations presented in this article. PKS 2005-489 has historically demonstrated a 100× dynamical range in the X-ray band. Thus, dramatically higher VHE fluxes $\left(10^{2}-10^{4} \times\right)$ can be expected in the future, unless such an increase in the X-ray flux is counter-balanced by a strong $(>10 \times)$ and simultaneous increase in the blazar's magnetic field. Further monitoring of this object is highly encouraged, as it is one of the few HBL easily detected at VHE during low states and has the potential for extreme brightness and variability.

The results presented here confirm the strong diagnostic potential of coordinated optical - X-ray - VHE observations. Future studies can be significantly improved by incorporating data from the recently launched Fermi $\gamma$-ray satellite. The 
Fermi data will provide information on the lower energy side of the inverse-Compton peak, enabling contemporaneous measurements of both sides of each blazar hump.

Acknowledgements. The support of the Namibian authorities and of the University of Namibia in facilitating the construction and operation of HESS is gratefully acknowledged, as is the support by the German Ministry for Education and Research (BMBF), the Max Planck Society, the French Ministry for Research, the CNRS-IN2P3 and the Astroparticle Interdisciplinary Programme of the CNRS, the UK Science and Technology Facilities Council (STFC), the IPNP of the Charles University, the Polish Ministry of Science and Higher Education, the South African Department of Science and Technology and National Research Foundation, and by the University of Namibia. We appreciate the excellent work of the technical support staff in Berlin, Durham, Hamburg, Heidelberg, Palaiseau, Paris, Saclay, and in Namibia in the construction and operation of the equipment. The article is based on observations obtained with XMM-Newton, an ESA science mission with instruments and contributions directly funded by ESA Member States and NASA. The authors thank the RXTE team for support during the ToO trigger. This research has made use of the NASA/IPAC Extragalactic Database (NED) which is operated by the Jet Propulsion Laboratory.

\section{References}

Abdo, A. A., Ackermann, M., Ajello, M., Atwood, W. B., et al. (LAT Collaboration) 2009, ApJ, 700, 597

Aharonian, F., Akhperjanian, A. G., Aye, K.-M., et al. (HESS Collaboration) 2004, Astroparticle Phys., 22, 109

Aharonian, F., Akhperjanian, A. G., Aye, K.-M., et al. (HESS Collaboration) 2005, A\&A, 436, L17

Aharonian, F., Akhperjanian, A. G., Bazer-Bachi, A. R., et al. (HESS Collaboration) 2006a, A\&A, 457, 899

Aharonian, F., Akhperjanian, A. G., Bazer-Bachi, A. R., et al. (HESS Collaboration) 2006b, A\&A, 448, L19

Aharonian, F., Akhperjanian, A. G., Bazer-Bachi, A. R., et al. (HESS Collaboration) 2006c, Nature, 440, 1018

Aharonian, F., Akhperjanian, A. G., Barres de Almeida, U., et al. (HESS Collaboration) 2007, A\&A, 475, L9

Aharonian, F., et al. (HESS Collaboration) 2009, A\&A, 502, 749

Baumgartner, W. H., \& Mushotzky, R. F. 2006, ApJ, 639, 929

Benbow, W. 2005, in Proceedings of Towards a Network of Atmospheric Cherenkov Detectors VII (Palaiseau), 163

Berge, D., Funk, S., \& Hinton, J. 2007, A\&A, 466, 1219

Cardelli, J. A., Clayton, G. C., \& Mathis, J. S. 1989, ApJ, 345, 245

Chadwick, P. M., Daniel, M. K., Lyons, K., et al. 2000, A\&A, 364, 450

Comastri, A., Fossati, G., Ghisellini, G., \& Molendi, S. 1997, ApJ, 480, 534

Coppi, P. S., \& Aharonian, F. A. 1999, ApJ, 521, L33

Dickey, J. M., \& Lockman, F. J. 1990, ARA\&A, 28, 215

Falomo, R., Maraschi, L., Treves, A., \& Tanzi, E. G. 1987, ApJ, 318, L39

Fazio, G. G., Ashby, M. L. N., Barmby, P., et al. 2004, ApJS, 154, 39

Feldman, G. J., \& Cousins, R. D. 1998, Phys. Rev. D, 57, 3873

Foschini, L., Treves, A., Tavecchio, F., et al. 2008, A\&A, 484, L35

Fossati, G., Buckley, J. H., Bond, I. H., et al. 2008, ApJ, 677, 906

Franceschini, A., Rodighiero, G., \& Vaccari, M. 2008, A\&A, 487, 837

Giommi, P., Barr, P., Pollock, A. M. T., Garilli, B., \& Maccagni, D. 1990, ApJ, 356,432

Giommi, P., \& Padovani, P. 1994, MNRAS, 268, L51

Hinton, J. A. 2004, New Astron. Rev., 48, 331

Jahoda, K., Swank, J. H., Giles, A. B., et al. 1996, in SPIE Conf. Ser. 2808, ed. O. H. Siegmund, \& M. A. Gummin, 59

Johnston, K. J., Fey, A. L., Zacharias, N., et al. 1995, AJ, 110, 880

Kalberla, P. M. W., Burton, W. B., Hartmann, D., et al. 2005, A\&A, 440, 775

Krawczynski, H., Hughes, S. B., Horan, D., et al. 2004, ApJ, 601, 151

Lamb, R. C., \& Macomb, D. J. 1997, ApJ, 488, 872

Li, T.-P., \& Ma, Y.-Q. 1983, ApJ, 272, 317

Lin, Y. C., Bertsch, D. L., Dingus, B. L., et al. 1996, ApJS, 105, 331

Madau, P., \& Pozzetti, L. 2000, MNRAS, 312, L9

Maraschi, L., Fossati, G., Tavecchio, F., et al. 1999, ApJ, 526, L81

Marshall, H. L., Fruscione, A., \& Carone, T. E. 1995, ApJ, 439, 90

Massaro, F., Tramacere, A., Cavaliere, A., Perri, M., \& Giommi, P. 2008, A\&A, 478,395

Nishijima, K. 2002, PASA, 19, 26

O’Donnell, J. E. 1994, ApJ, 422, 158
Padovani, P., Costamante, L., Giommi, P., et al. 2001, MNRAS, 328, 931 Perlman, E. S., Madejski, G., Stocke, J. T., \& Rector, T. A. 1999, ApJ, 523, L11 Pian, E., Vacanti, G., Tagliaferri, G., et al. 1998, ApJ, 492, L17

Roberts, M. D., Dazeley, S. A., Edwards, P. G., et al. 1998, A\&A, 337, 25

Roberts, M. D., McGee, P., Dazeley, S. A., et al. 1999, A\&A, 343, 691

Sambruna, R. M., Barr, P., Giommi, P., et al. 1994, ApJ, 434, 468

Sambruna, R. M., Urry, C. M., Ghisellini, G., \& Maraschi, L. 1995, ApJ, 449, 567

Scarpa, R., Urry, C. M., Falomo, R., Pesce, J. E., \& Treves, A. 2000, ApJ, 532, 740

Schlegel, D. J., Finkbeiner, D. P., \& Davis, M. 1998, ApJ, 500, 525

Silva, L., Granato, G. L., Bressan, A., \& Danese, L. 1998, ApJ, 509, 103

Stickel, M., Fried, J. W., Kuehr, H., Padovani, P., \& Urry, C. M. 1991, ApJ, 374, 431

Strüder, L., Briel, U., Dennerl, K., et al. 2001, A\&A, 365, L18

Tagliaferri, G., Ghisellini, G., Giommi, P., et al. 2000, A\&A, 354, 431

Tagliaferri, G., Ghisellini, G., Giommi, P., et al. 2001, A\&A, 368, 38

Tanihata, C., Urry, C. M., Takahashi, T., et al. 2001, ApJ, 563, 569

Turner, M. J. L., Abbey, A., Arnaud, M., et al. 2001, A\&A, 365, L27

Verner, D. A., Ferland, G. J., Korista, K. T., \& Yakovlev, D. G. 1996, ApJ, 465, 487

Wall, J. V., Shimmins, A. J., \& Bolton, J. G. 1975, Aust. J. Phys. Astrophys. Supp., 34, 55

Wall, J. V., Pettini, M., Danziger, I. J., Warwick, R. S., \& Wamsteker, W. 1986, MNRAS, 219, 23P

Wilms, J., Allen, A., \& McCray, R. 2000, ApJ, 542, 914

Zhang, Y. H. 2008, ApJ, 682, 789

1 Max-Planck-Institut für Kernphysik, PO Box 103980, 69029 Heidelberg, Germany

e-mail: luigi.costamante@stanford.edu;

wbenbow@cfa.harvard.edu

2 Yerevan Physics Institute, 2 Alikhanian Brothers St., 375036 Yerevan, Armenia

3 Centre d'Etude Spatiale des Rayonnements, CNRS/UPS, 9 Av. du Colonel Roche, BP 4346, 31029 Toulouse Cedex 4, France

4 Universität Hamburg, Institut für Experimentalphysik, Luruper Chaussee 149, 22761 Hamburg, Germany

5 Institut für Physik, Humboldt-Universität zu Berlin, Newtonstr. 15, 12489 Berlin, Germany

6 LUTH, Observatoire de Paris, CNRS, Université Paris Diderot, 5 Place Jules Janssen, 92190 Meudon, France

7 IRFU/DSM/CEA, CE Saclay, 91191 Gif-sur-Yvette, Cedex, France

8 University of Durham, Department of Physics, South Road, Durham DH1 3LE, UK

9 Unit for Space Physics, North-West University, Potchefstroom 2520, South Africa

10 Laboratoire Leprince-Ringuet, Ecole Polytechnique, CNRS/IN2P3, 91128 Palaiseau, France

11 Laboratoire d'Annecy-le-Vieux de Physique des Particules, Université de Savoie, CNRS/IN2P3, 74941 Annecy-le-Vieux, France

12 Astroparticule et Cosmologie (APC), CNRS, Université Paris 7 Denis Diderot, 10 rue Alice Domon et Léonie Duquet, 75205 Paris Cedex 13, France Fr $^{10}$

13 Dublin Institute for Advanced Studies, 5 Merrion Square, Dublin 2, Ireland

14 Landessternwarte, Universität Heidelberg, Königstuhl, 69117 Heidelberg, Germany

15 Laboratoire de Physique Théorique et Astroparticules, Université Montpellier 2, CNRS/IN2P3, CC 70, Place Eugène Bataillon, 34095 Montpellier Cedex 5, France

16 Universität Erlangen-Nürnberg, Physikalisches Institut, ErwinRommel-Str. 1, 91058 Erlangen, Germany

17 Laboratoire d'Astrophysique de Grenoble, INSU/CNRS, Université Joseph Fourier, BP 53, 38041 Grenoble Cedex 9, France

18 Institut für Astronomie und Astrophysik, Universität Tübingen, Sand 1, 72076 Tübingen, Germany

\footnotetext{
10 UMR 7164 (CNRS, Université Paris VII, CEA, Observatoire de Paris).
} 
The HESS Collaboration: VHE monitoring of PKS 2005-489 and simultaneous multi-wavelength observations

19 LPNHE, Université Pierre et Marie Curie Paris 6, Université Denis Diderot Paris 7, CNRS/IN2P3, 4 Place Jussieu, 75252, Paris Cedex 5, France

20 Charles University, Faculty of Mathematics and Physics, Institute of Particle and Nuclear Physics, V Holešovičkách 2, 180 00, Czechoslovakia

21 Institut für Theoretische Physik, Lehrstuhl IV: Weltraum und Astrophysik, Ruhr-Universität Bochum, 44780 Bochum, Germany

${ }^{22}$ University of Namibia, Private Bag 13301, Windhoek, Namibia

23 Obserwatorium Astronomiczne, Uniwersytet Jagielloński, ul. Orla 171, 30-244 Kraków, Poland

24 Nicolaus Copernicus Astronomical Center, ul. Bartycka 18, 00-716 Warsaw, Poland
25 School of Physics \& Astronomy, University of Leeds, Leeds LS2 9JT, UK

26 School of Chemistry \& Physics, University of Adelaide, Adelaide 5005, Australia

27 Toruń Centre for Astronomy, Nicolaus Copernicus University, ul. Gagarina 11, 87-100 Toruń, Poland

28 Instytut Fizyki Ja̧drowej PAN, ul. Radzikowskiego 152, 31-342 Kraków, Poland

29 Astronomical Observatory, The University of Warsaw, Al. Ujazdowskie 4, 00-478 Warsaw, Poland

30 European Associated Laboratory for Gamma-Ray Astronomy, jointly supported by CNRS and MPG 